\title{
ON INTERPOLATION AND APPROXIMATION BY RATIONAL FUNCTIONS WITH PREASSIGNED POLES*
}

\author{
BY \\ J. L. WALSH
}

1. Introduction. There has long been studied the problem of the approximation of analytic functions of a complex variable by polynomials, particularly with reference to (1) possibility of anproximating a given function by polynomials with an arbitrarily small $e_{i}$ ror, or of uniform expansion; (2) possibility of uniform expansion in a series of particular type, for instance polynomials found by interpolation, or polynomials belonging to a region; (3) degree of approximation, the study of the asymptotic behavior as $n$ becomes infinite of such a measure of approximation as

$$
\max \left[\left|f(z)-p_{n}(z)\right|, z \text { on } C\right]
$$

the measure of the approximation to the function $f(z)$ on a point set $C$ by the polynomial $p_{n}(z)$ of degiee $n$; (4) overconvergence, the phenomenon that a sequence approximating a given function $f(z)$ on a given point set $C$ frequently converges to the function $f(z)$ (or its analytic extension) not merely on $C$ but on a larger point set containing $C$ in its interior.

The problem of approximation of given functions not by polynomials but by more general rational functions has been less studied, but is of interest, not merely as a generalization of the problem of approximation by polynomials, but as a problem involving larger resources than the other, and whose study might be expected to be more fruitful. The properties of the sequences of rational functions depend largely on the positions of the poles of those functions, and such position plays an important rôle in the sequel. A judicious prescription of the position of the poles of the approximating rational functions or even lack of prescription of the poles may lead to more favorable results in (1)-(4) than prescription that the poles should lie at infinity.

The results of the present paper do not show, and are not intended to show, the usefulness in approximation of rational functions as contrasted with polynomials, namely in the problems mentioned. $\dagger$ Indeed, most of the new

* Presented to the Society, February 28, 1931; received by the editors May 28, 1931.

† Some results which bring out clearly this contrast are given by the present writer, Acta Mathematica, vol. 57 (1931), pp. 411- 435. 
material in the present paper which shows the advantage of rational functions over polynomials in connection with these problems (1)-(4) can readily be obtained from well known facts on approximation by polynomials by the use of linear transformations of the complex variable. The present paper does aim, however, to consider these problems (1)-(4) and to develop certain results on these topics which are common to very large classes of approximation by rational functions. Our purpose, then, is not to show the superiority of rational functions over polynomials for approximation, but rather to show that in spite of the apparent diversity of certain possible approximations by rational functions, these approximations still have many properties in common.

To be more explicit, we propose to study here interpolation and approximation to a given analytic function $f(z)$ by means of rational functions of the form

$$
f_{n}(z)=\frac{a_{0 n} z^{n}+a_{1 n} z^{n-1}+\cdots+a_{n n}}{\left(z-\alpha_{1 n}\right)\left(z-\alpha_{2 n}\right) \cdots\left(z-\alpha_{n n}\right)},
$$

where the $\alpha_{\text {in }}$ are prescribed and the $a_{i n}$ remain to be disposed of. The specific topics we discuss are the following. First we study approximation to the function $f(z)$ analytic for $|z| \leqq 1$ by the functions $f_{n}(z)$ where the $\alpha_{i n}$ have no limit point of modulus unity or less. Approximation is here measured in the sense of least squares, namely by the integral

$$
\int_{|z|=1}\left|f(z)-f_{n}(z)\right|^{2}|d z| \text {. }
$$

It turns out that for each $n$ the function $f_{n}(z)$ of best approximation is the function found by interpolation in the origin and in the $n$ points $1 / \bar{\alpha}_{i n}$, the inverses in the unit circle of the given points $\alpha_{i n}$. The convergence of this sequence $f_{n}(z)$ can be studied with reference to degree of approximation and overconvergence and yields some results on sequences of best approximation as measured by other methods, such as (1.1), or the surface integral

$$
\iint_{|z| \leqq 1}\left|f(z)-f_{n}(z)\right|^{p} d S, \quad p>0,
$$

or (1.2) where the exponent 2 is replaced by an arbitrary positive $p$. We next study the sequences of rational functions obtained by interpolation at the origin, at the $n$th roots of unity, and at points arbitrarily chosen. We also consider the specific case that the points $\alpha_{i n}$ are the points $\left(A^{n}\right)^{1 / n}$. Finally we add some remarks relative to approximation of an analytic function not on the unit circle but in an arbitrary Jordan region. 
Some of the methods we use are easy generalizations of the corresponding methods used for polynomial approximation, but others differ substantially from those previous methods.

The present study thus has connections with (1) the theory of the convergence of sequences of rational functions of best approximation, where the poles are preassigned, or are restricted to lie in certain given regions, or are entirely unrestricted; (2) the study of functions which can be represented by a series of the form $\sum A_{n} /\left(z-\alpha_{n}\right)$;* (3) Taylor's series, for our present discussion contains several different generalizations of Taylor's series. $\dagger$ Arbitrary analytic functions are approximated by rational functions with poles not necessarily at infinity, instead of by polynomials.

2. Approximation in the sense of least squares. We shall now prove the following theorem, which, together with its consequences, is our principal result:

THEOREM I. Let the function $f(z)$ be analytic for $|z| \leqq 1$ and let the numbers $\alpha_{i n}, i=1,2, \cdots, n ; n=1,2, \cdots$, be preassigned and have no limit point whose modulus is less than $A>1$. Denote by $f_{n}(z)$ the rational function of the form

$$
\begin{aligned}
f_{n}(z) & =A_{0 n}+\frac{A_{1 n}}{z-\alpha_{1 n}}+\frac{A_{2 n}}{z-\alpha_{2 n}}+\cdots+\frac{A_{n n}}{z-\alpha_{n n}} \\
& =\frac{a_{0 n} z^{n}+a_{1 n} z^{n-1}+\cdots+a_{n n}}{\left(z-\alpha_{1 n}\right)\left(z-\alpha_{2 n}\right) \cdots\left(z-\alpha_{n n}\right)}
\end{aligned}
$$

of best approximation to $f(z)$ on $C:|z|=1$ in the sense of least squares. $f$ Then the sequence $\left\{f_{n}(z)\right\}$ approaches the limit $f(z)$ uniformly for $|z| \leqq 1$. Moreover, if the function $f(z)$ is analytic for $|z|<T>1$, the sequence $\left\{f_{n}(z)\right\}$ approaches the limit $f(z)$ for $|z|<\left(A^{2} T+T+2 A\right) /\left(2 A T+A^{2}+1\right)$, uniformly for $|z|<R$ $<\left(A^{2} T+T+2 A\right) /\left(2 A T+A^{2}+1\right)$.

For the present we assume that the numbers $\alpha_{i n}$ for a given $n$ are all distinct; we shall later remove this restriction. We set $z=e^{i \theta}$ on the unit circle, so we are studying the best approximation to the given function $f(z)$ in the sense of least squares on the interval $0 \leqq \theta \leqq 2 \pi$ by the given functions 1 ,

* There is recent work by Wolff, Carleman, and Denjoy on this subject in continuation of the older work by Poincaré and Borel. For detailed references see Denjoy, Palermo Rendiconti, vol. 50 (1926), pp. 1-95.

$\dagger$ Various generalizations of Taylor's series in the complex domain have recently been given, particularly by Birkhoff, Widder, and the present writer. References are given by Widder, these Transactions, vol. 31 (1929), pp. 43--52.

$\ddagger$ The function of best approximation exists and is unique. See Walsh, these Transactions, vol. 33 (1931), pp. 668-689. 
$1 /\left(z-\alpha_{1 n}\right), 1 /\left(z-\alpha_{2 n}\right), \cdots, 1 /\left(z-\alpha_{n n}\right)$. The general formula for the linear combination of the linearly independent functions $f_{1}{ }^{0}(z), f_{2}{ }^{0}(z), \cdots, f_{m}{ }^{0}(z)$ which is the best approximation to $f(z)$ is*

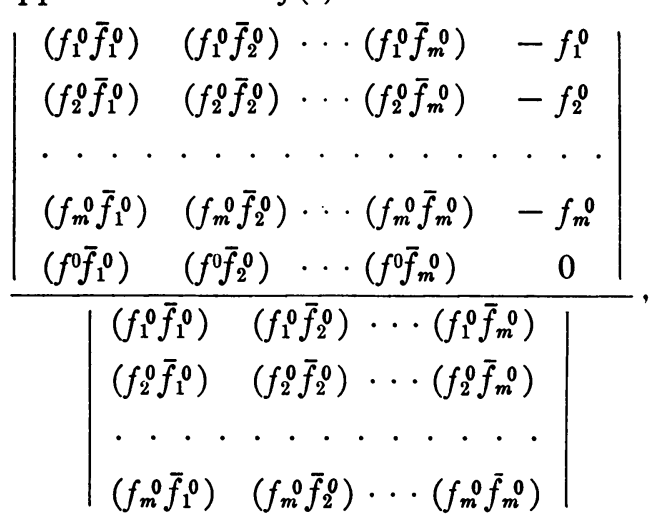

where we make use of the abbreviation

$$
\left(f_{i}^{0} \bar{f}_{i}^{0}\right)=\int_{0}^{2 \pi} f_{i}^{0}(z) \bar{f}_{i}^{0}(z) d \theta .
$$

By virtue of the relations $z=e^{i \theta}, d z=i z d \theta$, we have

$$
\begin{aligned}
\int_{z} \frac{1}{z-\alpha} \frac{d \theta}{\bar{z}-\bar{\alpha}^{\prime}} & =\int_{C} \frac{1}{z-\alpha} \frac{-\frac{i d z}{z}}{\frac{1}{z}-\bar{\alpha}^{\prime}} \\
& =i \int_{C} \frac{1}{z-\alpha} \frac{\frac{d z}{\bar{\alpha}^{\prime}}}{z-\frac{1}{\bar{\alpha}^{\prime}}}
\end{aligned}
$$

This expression is to be used only in case we have $|\alpha|,\left|\alpha^{\prime}\right|>1, \dagger$ so by Cauchy's integral formula the value of the integral is $-2 \pi /\left(1-\alpha \bar{\alpha}^{\prime}\right)$. We make the proper substitution in (2.2), setting $f_{1}{ }^{0}(z)=1, f_{2}{ }^{0}(z)=1 /\left(z-\alpha_{1 n}\right)$, $f_{2}{ }^{0}(z)=1 /\left(z-\alpha_{2 n}\right), \cdots, f_{n+1}{ }^{0}(z)=1 /\left(z-\alpha_{n n}\right)$. From each column of the determinants in the numerator and denominator we take the factor $2 \pi$, and then multiply the last row of the determinant in the numerator by $2 \pi$, so we obtain the following formula for the function of best approximation:

* Kowalewski, Determinantentheorie, Leipzig, 1909, p. 335.

$\dagger$ This inequality need not be satisfied by the $\alpha_{i n}$ for all values of $n$, but is surely satisfied if $n$ is sufficiently large. In the present paper we frequently write formulas which are valid only if $n$ is sufficiently large without explicit mention of that fact. 


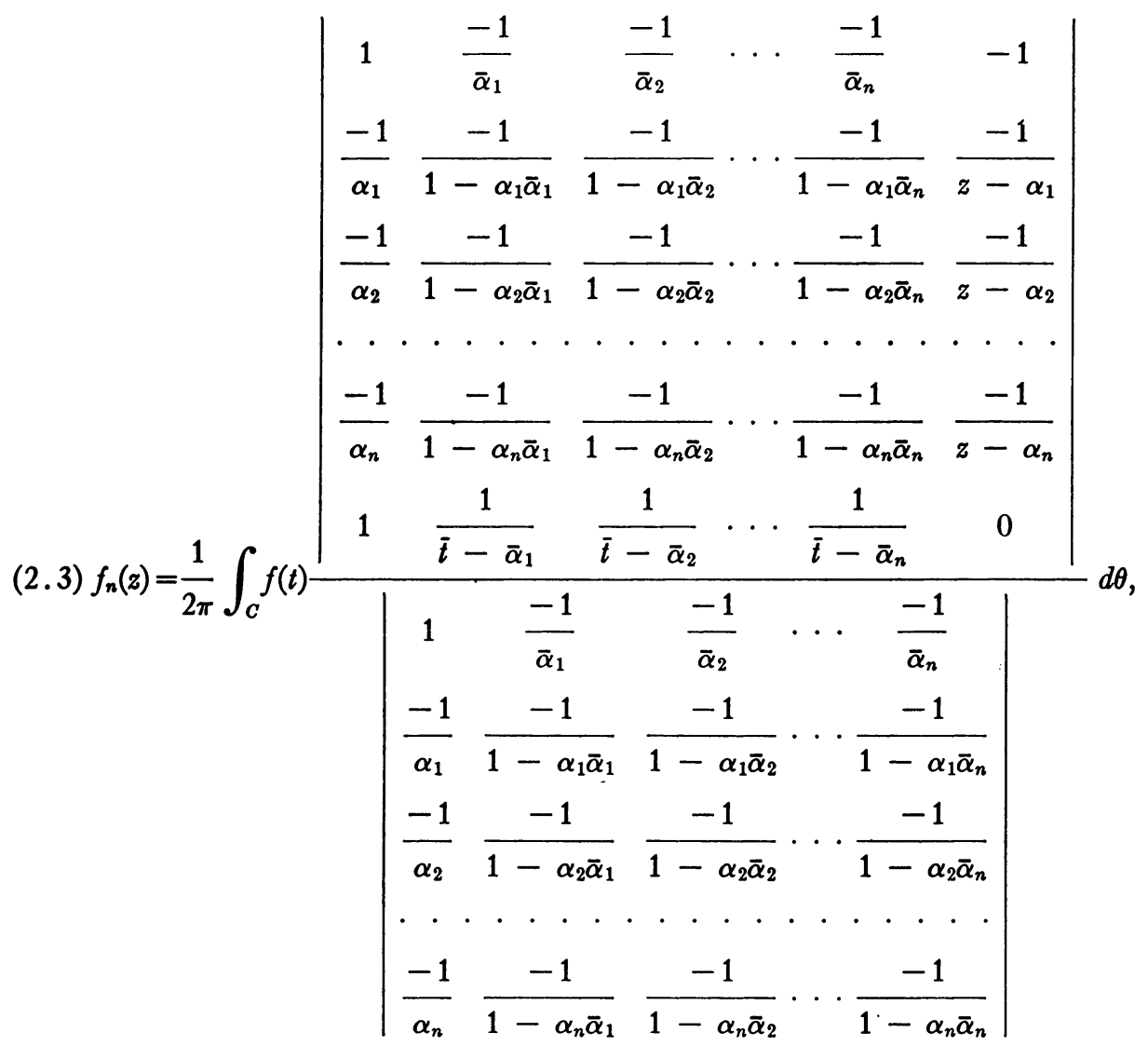

where integration is with respect to $\theta$, and $t=e^{i \theta}$. Here we have for simplicity omitted the second subscript (namely $n$ ) of the numbers $\alpha_{i}$. The denominator in (2.3) is different from zero. In fact the vanishing of the denominator in (2.2) is a necessary and sufficient condition that the functions $f_{1}{ }^{0}, f_{2}{ }^{0}, \cdots, f_{m}{ }^{0}$ should be linearly dependent, and the functions $f_{1}{ }^{0}, f_{2}{ }^{0}, \cdots, f_{m}{ }^{0}$ used in (2.3) are naturally linearly independent.

Let us here introduce Cauchy's formula

$$
\begin{aligned}
f(z) & =\frac{1}{2 \pi i} \int_{C} f(t) \frac{d t}{t-z} \\
& =\frac{1}{2 \pi} \int_{C} f(t) \frac{t d \theta}{t-z}
\end{aligned}
$$

and let us replace the $\bar{t}$ in (2.3) by its value $1 / t$, so that we have 


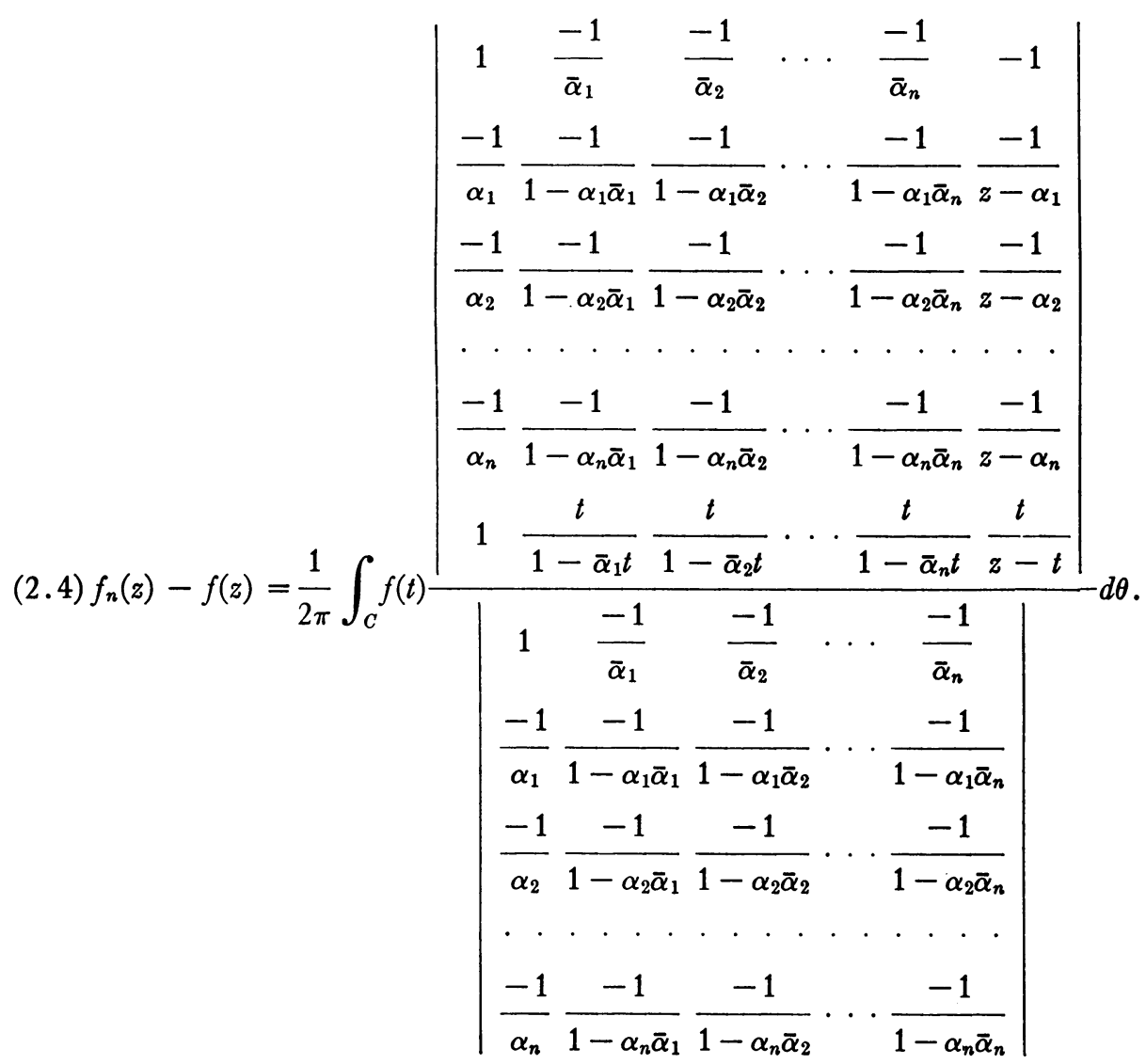

We prove that (2.4) simplifies to the form

$$
\begin{aligned}
& f_{n}(z)-f(z) \\
& =\frac{1}{2 \pi} \int_{C} f(t) \frac{z\left(\bar{\alpha}_{1} z-1\right)\left(\bar{\alpha}_{2} z-1\right) \cdots\left(\bar{\alpha}_{n} z-1\right)\left(t-\alpha_{1}\right)\left(t-\alpha_{2}\right) \cdots\left(t-\alpha_{n}\right)}{\left(z-\alpha_{1}\right)\left(z-\alpha_{2}\right) \cdots\left(z-\alpha_{n}\right)(z-t)\left(\bar{\alpha}_{1} t-1\right)\left(\bar{\alpha}_{2} t-1\right) \cdots\left(\bar{\alpha}_{n} t-1\right)} d \theta .
\end{aligned}
$$

Each determinant in (2.4) is to be evaluated by reducing each row of each determinant to a common denominator. The result (quotient of the original determinants) is a rational fraction whose denominator is the denominator which appears in (2.5). The proper numerator of (2.5) considered as a function of $z$ and $t$ is a polynomial in $z$ and $t$ which is seen by inspection of (2.4) to vanish whenever $z=0$ or $1 / \bar{\alpha}_{i}$, and likewise to vanish whenever $t=\alpha_{i}$. It remains to make sure that there is no other factor containing $z$ or $t$, and to evaluate the numerical factor.

The quotient in (2.4) is obviously of degree $n+1$ in $z$, hence can have no factor containing $z$ other than those in (2.5). The quotient in (2.4) is appar- 
ently of degree $n+2$ in $t$, but is in reality only of degree $n+1$. For the denominator in (2.5) is of degree $n+1$ in $t$, and when $t$ becomes infinite the quotient in (2.4) approaches zero, as is seen by inspection. There can therefore be no other factor in (2.5) which contains $z$ or $t$. Let us now take the iterated limit in the fraction of (2.4) as $z$ becomes infinite and then as $t$ becomes infinite. The resulting expression is seen by inspection to have the value unity, if the first row in the numerator is subtracted from the last row, so the equivalence of (2.4) and (2.5) is completely proved. It will be noticed too that (2.5) can be written

$$
\begin{aligned}
& f_{n}(z)-f(z) \\
& =\frac{1}{2 \pi i} \int_{C} f(t) \frac{z\left(\bar{\alpha}_{1} z-1\right)\left(\bar{\alpha}_{2} z-1\right) \cdots\left(\bar{\alpha}_{n} z-1\right)\left(t-\alpha_{1}\right)\left(t-\alpha_{2}\right) \cdots\left(t-\alpha_{n}\right)}{\left(z-\alpha_{1}\right)\left(z-\alpha_{2}\right) \cdots\left(z-\alpha_{n}\right)(z-t) t\left(\bar{\alpha}_{1} t-1\right)\left(\bar{\alpha}_{2} t-1\right) \cdots\left(\bar{\alpha}_{n} t-1\right)} d t,
\end{aligned}
$$

a form which involves the integral of an analytic function, so that the contour of integration may be deformed under suitable restrictions.

3. Proof of Theorem I. We are now in a position to prove, under the hypothesis of Theorem I, that the left-hand member of (2.6) approaches zero. We take the integral in (2.6) not over the circle $|t|=1$, but over the circle $C^{\prime}:|t|=T^{\prime}, 1<T^{\prime}<T$, where $f(z)$ is assumed analytic for $|z|<T$. Equation (2.6) is valid for $|z|<|t|=1$, and hence is valid for $|z|<|t|=T^{\prime}$. Let $A^{\prime}$ be an arbitrary number greater than unity and less than $A$. For $t$ on $C^{\prime}$ and for $n$ sufficiently large we have*

$$
\left|\frac{t-\alpha_{k}}{\bar{\alpha}_{k} t-1}\right|<\frac{T^{\prime}+A^{\prime}}{1+A^{\prime} T^{\prime}},
$$

and for $|z|=Z>1, Z<A^{\prime}$, we have

$$
\left|\frac{\bar{\alpha}_{k} z-1}{z-\alpha_{k}}\right|<\frac{A^{\prime} Z-1}{A^{\prime}-Z} .
$$

The left-hand member of (2.6) is, for $n$ sufficiently large, for $Z<T^{\prime}$, and for suitable choice of $M$, uniformly less than

$$
M\left(\frac{T^{\prime}+A^{\prime}}{1+A^{\prime} T^{\prime}} \frac{A^{\prime} Z-1}{A^{\prime}-Z}\right)^{n}
$$

where $M$ is independent of $n$, and this expression approaches zero provided

* This inequality and others which we shall use later are readily obtained by studying the transformations involved, in the present case $w=\left(t-\alpha_{k}\right) /\left(\bar{\alpha}_{k} t-1\right)$. In particular $|t|=1$ implies for the $w$ as just defined, $|w|=1$. 


$$
\frac{T^{\prime}+A^{\prime}}{1+A^{\prime} T^{\prime}} \frac{A^{\prime} Z-1}{A^{\prime}-Z}<1
$$

that is to say, provided

$$
Z<\frac{A^{\prime 2} T^{\prime}+T^{\prime}+2 A^{\prime}}{2 A^{\prime} T^{\prime}+A^{\prime 2}+1}
$$

this last quantity is less than $T^{\prime}$ and greater than unity. We have thus proved

$$
\lim _{n \rightarrow \infty} f_{n}(z)=f(z),
$$

uniformly for

$$
|z| \leqq R<\frac{A^{\prime 2} T^{\prime}+T^{\prime}+2 A^{\prime}}{2 A^{\prime} T^{\prime}+A^{\prime 2}+1} .
$$

The numbers $A^{\prime}<A$ and $T^{\prime}<T$ are arbitrary, so can be allowed to approach the limiting values $A$ and $T$ respectively. If this is done, the right-hand member of (3.2) increases, so (3.3) implies

$$
\lim _{n \rightarrow \infty} f_{n}(z)=f(\dot{z}),
$$

uniformly for

$$
|z| \leqq R<\frac{A^{2} T+T+2 A}{2 A T+A^{2}+1}
$$

In particular if $T=A$, this last expression reduces to

$$
\frac{A^{3}+3 A}{3 A^{2}+1}
$$

If, on the other hand, the function $f(z)$ is analytic at every finite point of the plane, we can allow $T^{\prime}$ to become infinite, and the corresponding expression in (3.4) becomes

$$
\frac{A^{2}+1}{2 A}
$$

If $T$ is arbitrary, and if we allow $A$ to become infinite, the expression in (3.4) approaches $T$ itself. Thus, if we have merely $\lim _{n \rightarrow \infty} \alpha_{i n}=\infty$ uniformly, we have convergence of $f_{n}(z)$ like that of Taylor's series, namely interior to an arbitrary circle $|z|<T>1$ within which $f(z)$ is analytic, uniform convergence for $|z| \leqq T^{\prime}<T$. This gives us in reality a generalization of Taylor's series; the various functions of the sequence are still rational functions not neces- 
sarily polynomials, but the character of their convergence both as to region of convergence and degree of convergence is like that of Taylor's series.

4. New derivation of formulas. It may seem that the discussion we have given is lacking in two respects: (1) the points $\alpha_{\text {in }}$ for each given $n$ have been assumed all distinct, (2) the points $\alpha_{i n}$ have been assumed finite. We now point out that those restrictions although necessary for the proofs as given are not necessary for the validity of the final formulas and other results.

The discussion about to be given is a derivation of equation (2.6) which is independent of the former derivation but which shows (2.6) to be valid even if the numbers $\alpha_{i n}$ for a given $n$ are not all distinct. The former derivation is to be considered useful as showing the relation between our present work and the classical formulas for approximation in the sense of least squares. We do not repeat that derivation in the more general case now to be considered because the notation necessary would be too complicated.

The function $f_{n}(z)$ is uniquely characterized by the properties (1) of being a rational function of degree $n$ with the prescribed poles, ${ }^{*}$ and (2) of being such that the function $f_{n}(z)-f(z)$ is orthogonal on $C$ to each of the given functions $1,1 /\left(z-\alpha_{k}\right)(k=1,2, \cdots, n)$, where if $p$ of the points $\alpha_{i n}$ (for a given $n$ ) coalesce say at $\alpha$, the function $f_{n}(z)-f(z)$ is orthogonal to each of the functions

$$
\frac{1}{z-\alpha}, \frac{1}{(z-\alpha)^{2}}, \cdots, \frac{1}{(z-\alpha)^{p}} .
$$

The first of these two properties is readily verified, for equation (2.6) may be written

$$
\begin{aligned}
& f_{n}(z)=\frac{1}{2 \pi i} \int_{C} f(t)\left[\frac{1}{t-z}\right. \\
& \left.-\frac{z\left(\bar{\alpha}_{1} z-1\right)\left(\bar{\alpha}_{2} z-1\right) \cdots\left(\bar{\alpha}_{n} z-1\right)\left(t-\alpha_{1}\right)\left(t-\alpha_{2}\right) \cdots\left(t-\alpha_{n}\right)}{\left(z-\alpha_{1}\right)\left(z-\alpha_{2}\right) \cdots\left(z-\alpha_{n}\right)(t-z) t\left(\bar{\alpha}_{1} t-1\right)\left(\bar{\alpha}_{2} t-1\right) \cdots\left(\bar{\alpha}_{n} t-1\right)}\right] d t .
\end{aligned}
$$

The two fractions in the bracket, when reduced to a common denominator, admit the factor $t-z$ in the numerator, for that numerator, considered as a polynomial in $t$ and $z$, vanishes for $t=z$. When the factor $t-z$ is cancelled, the new numerator is a polynomial in $t$ and $z$ of degree $n$ at most in $z$. The denominator is precisely $\left(z-\alpha_{1}\right)\left(z-\alpha_{2}\right) \cdots\left(z-\alpha_{n}\right)$, so far as factors containing $z$ are concerned. Hence the right-hand member of (4.2) actually is a rational function of the form prescribed in Theorem I. It remains to show that the function defined by (2.6) is orthogonal to the function $1 /\left(z-\alpha_{k}\right)$.

* We intend to imply by this phraseology merely that $f_{n}(z)$ can be written in the form of the last member of (2.1); we do not imply that the numerator and denominator have no common factor. 
Let us denote by $C^{\prime}$ a circle whose center is the origin, which lies interior to $C:|z|=1$, and which contains within it all the points $1 / \bar{\alpha}_{k}$. We have

$$
\begin{aligned}
& \int_{C}\left[f_{n}(z)-f(z)\right] \frac{d \theta}{\bar{z}-\bar{\alpha}_{k}}=i \int_{C}\left[f_{n}(z)-f(z)\right] \frac{d z}{\bar{\alpha}_{k} z-1} \\
= & i \int_{C^{\prime}}\left[f_{n}(z)-f(z)\right] \frac{d z}{\bar{\alpha}_{k} z-1}=\frac{1}{2 \pi} \int_{C^{\prime}} \frac{d z}{\bar{\alpha}_{k} z-1} \\
& \cdot \int_{C} f(t) \frac{z\left(\bar{\alpha}_{1} z-1\right)\left(\bar{\alpha}_{2} z-1\right) \cdots\left(\bar{\alpha}_{n} z-1\right)\left(t-\alpha_{1}\right)\left(t-\alpha_{2}\right) \cdots\left(t-\alpha_{n}\right)}{\left(z-\alpha_{1}\right)\left(z-\alpha_{2}\right) \cdots\left(z-\alpha_{n}\right)(z-t) t\left(\bar{\alpha}_{1} t-1\right)\left(\bar{\alpha}_{2} t-1\right) \cdots\left(\bar{\alpha}_{n} t-1\right)} d t \\
= & \frac{1}{2 \pi} \int_{C} f(t) \frac{\left(t-\alpha_{1}\right)\left(t-\alpha_{2}\right) \cdots\left(t-\alpha_{n}\right)}{t\left(\bar{\alpha}_{1} t-1\right)\left(\bar{\alpha}_{2} t-1\right) \cdots\left(\bar{\alpha}_{n} t-1\right)} d t \\
& \cdot \int_{C^{\prime}} \frac{z\left(\bar{\alpha}_{1} z-1\right) \cdots\left(\bar{\alpha}_{k-1} z-1\right)\left(\bar{\alpha}_{k+1} z-1\right) \cdots\left(\bar{\alpha}_{n} z-1\right)}{\left(z-\alpha_{1}\right)\left(z-\alpha_{2}\right) \cdots\left(z-\alpha_{n}\right)(z-t)} d z,
\end{aligned}
$$

and this last expression vanishes, for the integral over $C^{\prime}$ is zero by Cauchy's integral theorem.

We have shown merely that the function $f_{n}(z)-f(z)$ defined by $(2.6)$ is orthogonal on $C$ to the function $1 /\left(z-\alpha_{k}\right)$. It is entirely obvious that the proof can be modified so as to show that in case $p$ of the points $\alpha_{\text {in }}$ (for a given $n$ ) coalesce at $\alpha$, the function $f_{n}(z)-f(z)$ defined by (2.6) is orthogonal to each of the functions (4.1).

We leave to the reader the care of seeing that the function $f_{n}(z)-f(z)$ is orthogonal on $C$ to the function unity; the formulas already used require little modification.

There is no essential difficulty in modifying (2.6) so as to allow infinite values of the $\alpha_{i n}$. Thus if $\alpha_{1}=\infty$ for example, the fractions $\left(\bar{\alpha}_{1} z-1\right) /\left(z-\alpha_{1}\right)$ and $\left(t-\alpha_{1}\right) /\left(\bar{\alpha}_{1} t-1\right)$ are simply to be replaced by $-z$ and $-1 / t$ respectively, with these factors repeated if others of the $\alpha_{i}$ are infinite. In this latter case the functions (4.1) become $z, z^{2}, \cdots, z^{p}$, and these functions are orthogonal to the function $f_{n}(z)-f(z)$ defined by the modified (2.6).

All of the consequences, such as (3.4), which we have drawn from (2.6) are valid also in the new cases considered, namely that the points $\alpha_{i n}$ for a given $n$ are not necessarily all distinct, and that the point at infinity is admissible as one or more of the $\alpha_{i n}$.

5. Remarks on Theorem I. We add several other remarks in connection with Theorem I. Best approximation to $f(z)$ on $C$ in the sense of least squares by a rational function of the form (2.1) is equivalent to interpolation in the $n+1$ points $0,1 / \bar{\alpha}_{1}, 1 / \bar{\alpha}_{2}, \cdots, 1 / \bar{\alpha}_{n}$ interior to $C$, the inverses of the points $\infty, \alpha_{1}$, 
$\alpha_{2}, \cdots, \alpha_{n}$ with respect to $C$. For one sees by inspection that the right-hand member of (2.6) vanishes at the $n+1$ points enumerated, and the rational function $f_{n}(z)$ of form (2.1) which takes on given values in $n+1$ points is known to be unique. ${ }^{*}$ Coincidence of $p$ of these poles $\alpha_{i n}$ in $\alpha$ means coincidence of $p$ of the points of interpolation in $1 / \bar{\alpha}(p+1$ points if $\alpha=\infty)$, which means not merely equality of $f_{n}(1 / \bar{\alpha})$ and $f(1 / \bar{\alpha})$ but also of various derivatives of these two functions:

$$
f_{n}\left(\frac{1}{\bar{\alpha}}\right)=f\left(\frac{1}{\bar{\alpha}}\right), f_{n}^{\prime}\left(\frac{1}{\bar{\alpha}}\right)=f^{\prime}\left(\frac{1}{\bar{\alpha}}\right), \cdots, f_{n}^{(p-1)}\left(\frac{1}{\bar{\alpha}}\right)=f^{(p-1)}\left(\frac{1}{\bar{\alpha}}\right) .
$$

Taylor's series is well known to be found both by interpolation in the origin and by approximation on $C$ in the sense of least squares; this agrees with the result just found in the more general situation.

From (2.6) can be derived a result on the degree of convergence of the sequence $\left\{f_{n}(z)\right\}$. We are particularly interested in $z$ on $C$, and for this case we have $\left|\left(\bar{\alpha}_{k} z-1\right) /\left(z-\alpha_{k}\right)\right|=1$, so (compare (3.1)) for an arbitrary $R>(T+A) /(1+A T)$ and for a suitable $M^{\prime}$ depending on $R$ it follows that

$$
\left|f_{n}(z)-f(z)\right| \leqq M^{\prime} R^{n}, z \text { on } C .
$$

If the given function $f(z)$ is not assumed analytic on $C$, but merely analytic interior to $C$ and continuous $\dagger$ for $|z| \leqq 1$, our second derivation of (2.6) remains valid. In (2.6) we have $\left|\left(t-\alpha_{k}\right) /\left(\bar{\alpha}_{k} t-1\right)\right|=1$, and for $|z|=Z<1$ we have

$$
\left|\frac{\bar{\alpha}_{k} z-1}{z-\alpha_{k}}\right|<\frac{A Z+1}{A+Z}<1 .
$$

Hence we have $\lim _{n \rightarrow \infty} f_{n}(z)=f(z)$ uniformly for $|z| \leqq Z<1$.

The limit obtained in (3.4) is the best possible one; this means naturally best limit which holds for all admissible choices of $f(z)$ and the $\alpha_{i n}$. If all of the points $\alpha_{\text {in }}$ coincide at $z=A$, and if we approximate to the function $f(z)=$ $1 /(z+T)$, the approximating sequence $f_{n}(z)$ converges for

$$
|z|<\frac{A^{2} T+T+2 A}{2 A T+A^{2}+1}
$$

and converges throughout no concentric circle of larger radius.

* See for instance Walsh, these Transactions, vol. 33 (1931), pp. 668-689.

† Indeed it is sufficient if $f(z)$ as defined on $C$ is merely integrable together with its square. This is independent of the consideration of $f(z)$ as the boundary value of an analytic function. The limit of the sequence $f_{n}(z)$ for $|z|<1$ is then defined by

$$
\frac{1}{2 \pi i} \int_{C} \frac{f(t) d t}{t-z}
$$


The functions $f_{n}(z)$ can be written down by inspection, from their form (2.1) and from the fact that they coincide with $f(z)$ in the origin and satisfy the equations

$$
f_{n}^{(k)}(1 / A)=f^{(k)}(1 / A) \quad(k=0,1, \cdots, n-1) .
$$

We have

$$
f(z)-f_{n}(z)=-\frac{(T+A)^{n} z(A z-1)^{n}}{T(A T+1)^{n}(z+T)(z-A)^{n}} .
$$

For the particular value $z=\left(A^{2} T+T+2 A\right) /\left(2 A T+A^{2}+1\right)$, we have

$$
\frac{(T+A)(A z-1)}{(A T+1)(z-A)}=-1,
$$

and hence the right-hand member of (5.1) approaches no limit.

6. The limit points of the $\alpha_{i n}$ restricted to an arbitrary circular region. The problem we have been considering, approximation to $f(z)$ on $C$ in the sense of least squares, is, as we have seen, equivalent to interpolation in the origin as well as in the points $1 / \bar{\alpha}_{i}$. We now show that the results on convergence obtained in $\S 3$ are likewise valid if we choose an arbitrary point $\beta$ interior to $C$ but which may depend on $n$, and interpolate in $\beta$ instead of in the origin, provided merely that $1-|\beta|$ remains greater than some positive quantity as $n$ becomes infinite. Let us denote by $f_{n}{ }^{0}(z)$ the approximating function of degree $n$, with poles in the points $\alpha_{i n}$, which coincides with $f(z)$ in the points $\beta, 1 / \bar{\alpha}_{1}, 1 / \bar{\alpha}_{2}, \cdots, 1 / \bar{\alpha}_{n}$. We have

$$
\begin{aligned}
& f_{n}^{0}(z)-f(z) \\
& =\frac{1}{2 \pi i} \int_{C} f(t) \frac{(z-\beta)\left(\bar{\alpha}_{1} z-1\right) \cdots\left(\bar{\alpha}_{n} z-1\right)\left(t-\alpha_{1}\right) \cdots\left(t-\alpha_{n}\right)}{\left(z-\alpha_{1}\right) \cdots\left(z-\alpha_{n}\right)(z-t)(t-\beta)\left(\bar{\alpha}_{1} t-1\right) \cdots\left(\bar{\alpha}_{n} t-1\right)} d t,
\end{aligned}
$$

for the function $f_{n}{ }^{0}(z)$ defined by (6.1) clearly coincides with $f(z)$ in the prescribed $n+1$ points, and the function $f_{n}{ }^{0}(z)$ thus defined is related to $f_{n}(z)$ by the equation (found from (2.6))

(6.2) $f_{n}^{0}(z)-f_{n}(z)=\frac{1}{2 \pi i} \int_{C} f(t) \frac{\beta\left(\bar{\alpha}_{1} z-1\right) \cdots\left(\bar{\alpha}_{n} z-1\right)\left(t-\alpha_{1}\right) \cdots\left(t-\alpha_{n}\right)}{\left(z-\alpha_{1}\right) \cdots\left(z-\alpha_{n}\right) t(t-\beta)\left(\bar{\alpha}_{1} t-1\right) \cdots\left(\bar{\alpha}_{n} t-1\right)} d t$.

The right-hand member of (6.2) is a rational function of degree $n$ with poles only in the prescribed $n$ points, and hence $f_{n}{ }^{0}(z)$ is also such a function. Moreover it is clear by inspection of (6.1) and of the discussion in $\$ 3$ that in (3.4) we can replace $f_{n}(z)$ by $f_{n}{ }^{0}(z)$. Convergence of the sequence $f_{n}{ }^{0}(z)$ is proved under the restrictions previously found for $f_{n}(z)$; the verification of this fact is left to the reader. 
We use the term circular region to denote the closed interior or exterior of a circle, or a (closed) half-plane, and we use the same notation for a circular region as for its boundary. We shall study a problem more general than that of Theorem I, where now the points $\alpha_{i n}$ are assumed to have no limit point exterior to an arbitrary circular region $\Gamma$ which has no point in common with $C$. Let us transform the circle $\Gamma$ into a circle $\Gamma^{\prime}$ concentric with $C$, by a transformation of the form $w=(z-\beta) /(-1+\bar{\beta} z)$, which carries $C$ and its interior into $C$ and its interior; it is sufficient to choose $\beta$ interior to $C$ and one of the two points mutually inverse in both $C$ and $\Gamma$. Approximating $f(z)$ on $C$ in the sense of least squares is equivalent to interpolation of $f(z)$ in the points $z=0,1 / \bar{\alpha}_{k}$. A rational function of degree $n$ in $z$ is a rational function of degree $n$ in $w$, and if the former has its poles in the points $\alpha_{k}$, the latter has its poles in the corresponding points $w=\left(\alpha_{k}-\beta\right) /\left(-1+\bar{\beta} \alpha_{k}\right)$. Interpolation in the points $w=0$ and in the points $w=\left(\beta \bar{\alpha}_{k}-1\right) /\left(\bar{\alpha}_{k}-\bar{\beta}\right)$ is equivalent to approximation to $f(z)$ on $C$ in the sense of least squares, for which we have obtained the results of Theorem I, and if we replace $w=0$ by the point $w=\beta$ which corresponds to $z=0$ we have equivalent results on convergence (as has just been proved) valid then for our original problem in the $z$-plane. The points $w=\left(\beta \bar{\alpha}_{k}-1\right) /\left(\bar{\alpha}_{k}-\bar{\beta}\right)$ are not only the inverses with respect to $C$ of the points $w=\left(\alpha_{k}-\beta\right) /\left(-1+\bar{\beta} \alpha_{k}\right)$, but are also the transforms of the points $z=1 / \bar{\alpha}_{k}$.

In order to interpret these results in the $z$-plane, we write the equation of the circle $\Gamma$ in the form

$$
\left|\frac{z-\beta}{\bar{\beta} z-1}\right|=A>1,
$$

where, as we have said, $\beta$ is interior to $C$, and where $\beta$ and $1 / \bar{\beta}$ are mutually inverse points with respect to both $C$ and $\Gamma$. It is naturally possible to write $\Gamma$ in the form (6.3), for $\Gamma$ belongs to the coaxial family of circles determined by $\beta$ and $1 / \bar{\beta}$ as null-circles. We have therefore proved

THEOREM II. Let the function $f(z)$ be analytic for $|(z-\beta) /(\bar{\beta} z-1)|<T>1$, where $|\beta|<1$, and let the points $\alpha_{\text {in }}$ have no limit point $z$ for which $\mid(z-\beta) /(\bar{\beta} z$ $-1) \mid<A>1$. Then the sequence $\left\{f_{n}(z)\right\}$ of rational functions of respective degrees $n$ with poles in the points $\alpha_{\text {in }}$ of best approximation to $f(z)$ on $C:|z|=1$ in the sense of least squares converges to the function $f(z)$ whenever we have

$$
\left|\frac{z-\beta}{\bar{\beta} z-1}\right|<\frac{A^{2} T+T+2 A}{2 A T+A^{2}+1},
$$

uniformly whenever we have 


$$
\left|\frac{z-\beta}{\bar{\beta} z-1}\right|<R<\frac{A^{2} T+T+2 A}{2 A T+A^{2}+1} .
$$

The circular region

$$
\left|\frac{z-\beta}{\bar{\beta} z-1}\right| \leqq R
$$

is the interior of a circle, a half-plane, or the exterior of a circle according as $R$ is less than, equal to, or greater than $1 /|\beta|$.

It is a corollary of Theorem II that if $\lim _{n \rightarrow \infty} \alpha_{i n}=1 / \bar{\beta}$ uniformly with respect to $i$, then the sequence $\left\{f_{n}(z)\right\}$ converges for $|(z-\beta) /(\bar{\beta} z-1)|<T$, uniformly for $|(z-\beta) /(\bar{\beta} z-1)|<R<T$. Theorem $\mathrm{I}$ is the special case of Theorem II corresponding to $\beta=0$.

7. Points $\alpha_{\text {in }}$ approaching $C$. We have hitherto assumed the points $\alpha_{\text {in }}$ to have no limit point on $C$. We shall now study the convergence of the sequence $f_{n}(z)$ of best approximation in the sense of least squares where this restriction is removed; we naturally still require $f(z)$ to be analytic on and within $C$. If $f(z)$ is analytic for $|z| \leqq 1$, or is merely analytic for $|z|<1$ and continuous for $|z| \leqq 1$, and if we have merely $\left|\alpha_{i n}\right| \geqq A>1$, the sequence $f_{n}(z)$ converges to the value $f(z)$ for $|z|<1$, uniformly for $|z| \leqq Z<1$. This follows directly by the method used in $\$ 3$, where the integral in (2.6) is taken over $C$, so that we have

$$
\left|\frac{t-\alpha_{k}}{\bar{\alpha}_{k} t-1}\right|=1, t \text { on } C ;\left|\frac{\bar{\alpha}_{k} z-1}{z-\alpha_{k}}\right| \leqq \frac{A Z+1}{A+Z}<1,|z| \leqq Z<1 .
$$

The question of uniform convergence for $|z| \leqq 1$ of $f_{n}(z)$ still remains, when $f(z)$ is analytic for $|z| \leqq 1$, and when the quantities $\left|\alpha_{i n}\right|$ are not bounded from unity as $n$ becomes infinite; this is the question we now discuss. Our present hypothesis is $\left|\alpha_{i n}\right| \geqq A_{n}>1$, and we employ again the method of §3. The integral in (2.6) can be taken over a circle $|t|=T^{\prime}, 1<T^{\prime}<T$. For $z$ on $C$ we have

so our proof that

$$
\left|\frac{\bar{\alpha}_{k} z-1}{z-\alpha_{k}}\right|=1
$$

$$
\lim _{n \rightarrow \infty} f_{n}(z)=f(z) \text { uniformly for }|z| \leqq 1
$$

will be complete provided we have

$$
\lim _{n \rightarrow \infty}\left(\frac{T^{\prime}+A_{n}}{1+A_{n} T^{\prime}}\right)^{n}=0
$$


THEOREM III. If the function $f(z)$ is analytic for $|z|<T>1$, and if we have $\left|\alpha_{\text {in }}\right| \geqq A_{n}>1$, the sequence of rational functions $f_{n}(z)$ of best approximation to $f(z)$ on $C:|z|=1$ in the sense of least squares converges to the value $f(z)$ uniformly for $|z| \leqq 1$ provided that

$$
\lim _{n \rightarrow \infty} n\left(A_{n}-1\right)=\infty
$$

We shall shortly identify condition (7.3) with condition (7.2). Condition (7.3) is obviously not satisfied if we have

$$
A_{n}=1+\frac{\kappa}{n}, \kappa \text { independent of } n \text {. }
$$

In this case one finds

$$
\left(\frac{T^{\prime}+A_{n}}{1+A_{n} T^{\prime}}\right)^{n}=\left(\frac{T^{\prime}+1+\kappa / n}{1+T^{\prime}+\kappa T^{\prime} / n}\right)^{n}=\left(\frac{1+\frac{\kappa}{\left(1+T^{\prime}\right) n}}{1+\frac{\kappa T^{\prime}}{\left(1+T^{\prime}\right) n}}\right)^{n},
$$

which approaches the value $e^{x /\left(1+T^{\prime}\right) / e^{x} T^{\prime} /\left(1+T^{\prime}\right)}$. On the other hand, condition (7.3) is obviously satisfied for every $T^{\prime}$ if we have

$$
A_{n} \geqq 1+\frac{\kappa}{n^{\lambda}}, 0<\lambda<1, \kappa \text { independent of } n
$$

In Theorem III we are primarily concerned with the case that either the numbers $A_{n}$ or an infinite sequence of them approach the value unity. This condition is, as a matter of fact, not essential for the truth of that theorem. We shall find it convenient to assume in the sequel

$$
\lim _{n \rightarrow \infty} A_{n}=1
$$

but that is purely a matter of convenience.

We have

$$
n \log \left(\frac{T^{\prime}+A_{n}}{1+A_{n} T^{\prime}}\right)=n \log \left[1-\frac{\left(T^{\prime}-1\right)\left(A_{n}-1\right)}{1+A_{n} T^{\prime}}\right]
$$

and we compare this equation with 


$$
\begin{aligned}
n \log (1-x) & =-n x-n x^{2}\left[\frac{1}{2}+\frac{x}{3}+\frac{x^{2}}{4}+\cdots\right],|x|<1, \\
x & =\frac{\left(T^{\prime}-1\right)\left(A_{n}-1\right)}{1+A_{n} T^{\prime}}>0 .
\end{aligned}
$$

If (7.3) is satisfied, we see by inspection that the right-hand member of (7.5) becomes negatively infinite, and hence (7.2) is fulfilled. On the other hand, if $n\left(A_{n}-1\right)$ does not become infinite, $n\left(A_{n}-1\right)$ is uniformly bounded for a certain sequence $n_{1}, n_{2}, \cdots$ of indices $n$. For this same sequence of indices the square bracket in (7.5) approaches the value $1 / 2$, the expression $n x^{2}$ approaches the value zero, and condition (7.2) fails to be satisfied. Thus (7.2) and (7.3) are shown to be completely equivalent; condition (7.2) is independent of $T^{\prime}>1$; in Theorem III we use the fact that (7.3) implies (7.2), and Theorem III is now completely proved.

Condition (7.3) (or condition (7.2)) might seem artificial, and it might be supposed that this condition were merely a convenient sufficient condition for the conclusion of Theorem III. But we can show that condition (7.3) can be replaced by no weaker condition and still imply the conclusion of Theorem III for all functions $f(z)$ which satisfy the hypothesis. We show this by means of the example

$$
f(z)=\frac{1}{z+T}, \quad \alpha_{i n}=A_{n} ; T>1, A_{n}>1 .
$$

We can write down by inspection a rational function $f_{n}(z)$ with all of its poles in the point $A_{n}$, which agrees with the function $f(z)$ at the origin, and which together with its first $n-1$ derivatives agrees with $f(z)$ and its first $n-1$ derivatives at the point $z=1 / A_{n}$. This function $f_{n}(z)$ is uniquely determined by these conditions, and is known to be the admissible function of best approximation to $f(z)$ on $C$ in the sense of least squares. We have

$$
f(z)-f_{n}(z)=-\frac{\left(T+A_{n}\right)^{n} z\left(A_{n} z-1\right)^{n}}{T\left(A_{n} T+1\right)^{n}(z+T)\left(z-A_{n}\right)^{n}},
$$

as the reader may verify. For $z$ on $C$, it appears that

$$
\left|f(z)-f_{n}(z)\right|=\frac{1}{T|z+T|}\left|\frac{T+A_{n}}{1+A_{n} T}\right|^{n},
$$

so that for the particular function $f(z)=1 /(z+T)$, equation (7.2) or (7.3) is a necessary and sufficient condition that $f_{n}(z)$ should approach $f(z)$ at even a single point of $C$.

Theorem III is concerned with the uniform convergence on $C$ of a particu- 
lar sequence of functions, namely the best approximation to $f(z)$ on $C$ in the sense of least squares. One might ask whether some other sequence $F_{n}(z)$ of functions (of respective degrees $n$ and with the prescribed poles) can converge to $f(z)$ uniformly for $|z|=1$ even when condition (7.3) is not fulfilled. It is still true that condition (7.3) can be replaced by no weaker condition which implies $\lim _{n \rightarrow \infty} F_{n}(z)=f(z)$ uniformly on $C$ for all functions $f(z)$ which satisfy the hypothesis of Theorem III. If we had

$$
\lim _{n \rightarrow \infty} F_{n}(z)=f(z), \text { uniformly for } z \text { on } C,
$$

for the particular function $f(z)=1 /(z+T)$ already considered, we should have also

$$
\lim _{n \rightarrow \infty} \int_{C}\left|f(z)-F_{n}(z)\right|^{2}|d z|=0 .
$$

This contradicts the inequality

$$
\int_{C}\left|f(z)-f_{n}(z)\right|^{2}|d z| \leqq \int_{C}\left|f(z)-F_{n}(z)\right|^{2}|d| z,
$$

found from the definition of the $f_{n}(z)$ (i.e. functions of best approximation in the sense of least squares), and the inequality

$$
\int_{C}\left|f(z)-f_{n}(z)\right|^{2}|d z| \geqq \frac{2 \pi}{T^{2}(T-1)^{2}}\left|\frac{T+A_{n}}{1+A_{n} T}\right|^{2 n}
$$

derived from (7.6).

Indeed, the material just given proves that $\lim _{k \rightarrow \infty} F_{n_{k}}(z)=f(z)$ uniformly for $z$ on $C$ for $f(z)=1 /(z+T)$ is impossible unless the condition (7.3) or (7.2) is satisfied for this particular sequence $n_{k}$.

Condition (7.3) is derived on the assumption $\left|\alpha_{i n}\right| \geqq A_{n}$, and our remarks on the generality of that condition are based on the maintaining of that assumption. There can naturally be derived a more general although slightly less simple condition involving the precise quantities $\left|\alpha_{i n}\right|=A_{i n}$, where the above assumption is allowed to fall.

If $f(z)$ and $f_{n}(z)$ have the same meaning as in Theorem III, a necessary and sufficient condition that we have

$$
\lim _{n \rightarrow \infty} f_{n}(z)=f(z), \text { uniformly for }|z| \leqq 1,
$$

for every choice of the function $f(z)$ analytic for $|z|<T>1$, and for every choice of $\alpha_{i n},\left|\alpha_{i n}\right|=A_{i n}$, is 


$$
\lim _{n \rightarrow \infty} \sum_{i=1}^{n}\left(\frac{A_{\text {in }}-1}{A_{\text {in }}}\right)=\infty \text {. }
$$

From the integral formula for $f_{n}(z)-f(z)$ it is seen as before that the condition

$$
\lim _{n \rightarrow \infty} \prod_{i=1}^{n} \frac{T^{\prime}+A_{\text {in }}}{1+A_{\text {in }} T^{\prime}}=0
$$

is sufficient. This condition is also necessary, as the reader will verify by the choice $\alpha_{i n}=A_{i n}, f(z)=1 /(z+T)$; the formulas are almost identical with those already used. Condition (7.8) is, as will appear, independent of the choice of $T^{\prime}$.

Let us prove that conditions (7.7) and (7.8) are completely equivalent. Condition (7.7) is equivalent to the condition

$$
\lim _{n \rightarrow \infty} \sum_{i=1}^{n} \frac{(T-1)\left(A_{i n}-1\right)}{1+A_{i n} T}=\infty,
$$

for we have by $A_{\text {in }}>1$

$$
\frac{T-1}{T+1} \leqq \frac{\frac{(T-1)\left(A_{\text {in }}-1\right)}{1+A_{\text {in }} T}}{\frac{A_{\text {in }}-1}{A_{\text {in }}}} \leqq \frac{T-1}{T} .
$$

For $0<x \leqq X<1$ the condition

$$
m x<\log (1-x)<M x, m<0, M<0,
$$

is satisfied for suitable values of $m$ and $M$. The quantity $(T-1)\left(A_{\text {in }}-1\right) /(1$ $\left.+4 A_{i n} T\right)$ is positive and less than $(T-1) / T$, so condition (7.9) is equivalent to

$$
\lim _{n \rightarrow \infty} \sum_{i=1}^{n} \log \left(1-\frac{(T-1)\left(A_{i n}-1\right)}{1+A_{i n} T}\right)=\lim _{n \rightarrow \infty} \sum_{i=1}^{n} \log \left(\frac{T+A_{i n}}{1+A_{\text {in }} T}\right)=-\infty \text {, }
$$

and this condition, being independent of $T$, is equivalent to (7.8). The proof is now complete.

Condition (7.3) obviously implies (7.7), if $A_{i n} \geqq A_{n}$, for we need consider only the case $\lim _{n \rightarrow \infty} A_{n}=1$. If $A_{n}<2$ we have

$$
\begin{gathered}
\frac{A_{\text {in }}-1}{A_{\text {in }}} \geqq \frac{A_{n}-1}{A_{n}}>\frac{A_{n}-1}{2}, \\
2 \sum_{i=1}^{n}\left(\frac{A_{\text {in }}-1}{A_{\text {in }}}\right)>n\left(A_{n}-1\right) .
\end{gathered}
$$

If the latter quantity becomes infinite, so also does the former. 
Condition (7.3) can also be written in the form

$$
\lim _{n \rightarrow \infty} A_{n}^{n}=\infty \text {. }
$$

It is sufficient to consider the case $\lim _{n \rightarrow \infty} A_{n}=1$. An inequality

$$
m x<\log (1+x)<M x, \quad m, M>0,
$$

is valid for suitably chosen $m$ and $M$ for sufficiently small positive $x$. Hence condition (7.3) is the same as

$$
n \log \left[1+\left(A_{n}-1\right)\right] \rightarrow \infty
$$

which is another form for (7.10).

Condition (7.7) can also be written in a simpler form, namely,

$$
\lim _{n \rightarrow \infty} \sum_{i=1}^{n}\left(A_{i n}-1\right)=\infty
$$

provided that the numbers $A_{\text {in }}$ are uniformly limited, $A_{\text {in }} \leqq A^{\prime}$.

8. Other measures of approximation. There are interesting measures of approximation other than least squares, for instance (1) that of Tchebycheff (already described in connection with (1.1)) taken on $C:|z|=1$, (2) least weighted $p$ th powers on the circumference $C$, (3) least weighted $p$ th powers measured over the area $|z| \leqq 1$. We shall treat these cases separately, and shall prove in each case

TheOREM IV. If the numbers $\alpha_{\text {in }}$ have no limit point whose modulus is less than $A>1$, and if the function $f(z)$ is analytic for $|z|<T>1$, then the sequence $\left\{F_{n}(z)\right\}$ of rational functions of respective degrees $n$ with poles $\alpha_{i n}$ of best approximation to $f(z)$ converges to the limit $f(z)$ for

$$
|z|<\frac{A^{2} T+T+2 A}{2 A T+A^{2}+1} \text {, uniformly for }|z| \leqq R<\frac{A^{2} T+T+2 A}{2 A T+A^{2}+1} .
$$

Let us first treat case (1); the sequence of rational functions of best approximation exists and is unique. ${ }^{*}$ If $R$ is arbitrary, but greater than $(T+A) /(1+A T)$, the inequality

$$
\left|f_{n}(z)-f(z)\right| \leqq M^{\prime} R^{n}, z \text { on } C,
$$

is proved in $\S 5$, where the functions $f_{n}(z)$ are the rational functions studied in

* Walsh, these Transactions, vol. 33 (1931), pp. 668-689.

Case (1) is equivalent to approximation on $|z| \leqq 1$ in the sense of Tchebycheff. These two cases may also be extended to include approximation in the sense of Tchebycheff with a norm function; the modifications are left to the reader. 
Theorem I. Inequality (8.1), holding for the functions $f_{n}(z)$, must also hold for the functions $F_{n}(z)$ of best approximation in the sense of Tchebycheff:

$$
\left|F_{n}(z)-f(z)\right| \leqq M^{\prime} R^{n}, z \text { on } C .
$$

By combining (8.1) and (8.2) we obtain

$$
\left|f_{n}(z)-F_{n}(z)\right| \leqq 2 M^{\prime} R^{n}, z \text { on } C ;
$$

the function whose absolute value appears here is a rational function of degree $n$.

We now make use of the following lemma:*

LemMa I. If $P(z)$ is a rational function of degree $n$ whose poles lie on or exterior to the circle $|z|=\lambda \rho>\lambda$, and if we have

then we have

$$
|P(z)| \leqq L, \text { for }|z|=\lambda
$$

$$
|P(z)| \leqq L\left(\frac{\rho R_{1}-1}{\rho-R_{1}}\right)^{n}, \text { for }|z|=\lambda R_{1}, 1<R_{1}<\rho .
$$

Under the present circumstances, we have, by (8.3) and (8.4),

$$
\left|f_{n}(z)-F_{n}(z)\right| \leqq 2 M^{\prime} R^{n}\left(\frac{A^{\prime} R_{1}-1}{A^{\prime}-R_{1}}\right)^{n},|z| \leqq R_{1}<A^{\prime}<A .
$$

That is to say, the sequence $\left\{f_{n}(z)-F_{n}(z)\right\}$ converges for $|z| \leqq R_{1}$ provided

$$
R\left(\frac{A^{\prime} R_{1}-1}{A^{\prime}-R_{1}}\right)<1 \text {, or } R_{1}<\frac{A^{\prime}+R}{1+A^{\prime} R} .
$$

The number $R$ is here arbitrary, greater than $(T+A) /(1+A T)$, and $A^{\prime}$ is arbitrary less than $A$, so the sequence $\left\{f_{n}(z)-F_{n}(z)\right\}$ converges for

$$
|z|<\frac{A T^{2}+T+2 A}{2 A T+A^{2}+1} \text {, uniformly for }|z| \leqq R_{1}^{\prime}<\frac{A T^{2}+T+2 A}{2 A T+A^{2}+1} \text {. }
$$

The sequence $\left\{f_{n}(z)\right\}$ converges (Theorem I) under these restrictions on $z$, hence the sequence $\left\{F_{n}(z)\right\}$ does also, and Theorem IV is proved in case (1).

In case (2) we are dealing with the sequence $\left\{F_{n}(z)\right\}$ of best approximation to $f(z)$ on $C$ in the sense of least weighted $p$ th powers as measured on $C$, that is, with the sequence of rational functions $F_{n}(z)$ of respective degrees $n$ and having their poles in the prescribed points such that

$$
\int_{C}\left|F_{n}(z)-f(z)\right| p_{n}(z)|d z|, p>0
$$

* Walsh, these Transactions, vol. 30 (1928), pp. 838-847; p. 842. 
is not greater than the corresponding integral for any other rational function of degree $n$ whose poles lie in the prescribed points. The norm function $n(z)$ is supposed to be positive and continuous on $C$, and under these circumstances the function of best approximation always exists, and is unique if $p>1$.

Denote by $n^{\prime}$ and $n^{\prime \prime}$ two numbers such that we have

$$
0<n^{\prime}<n(z)<n^{\prime \prime}, z \text { on } C .
$$

For the functions $f_{n}(z)$ of Theorem I we have by (8.1)

$$
\int_{C}\left|f_{n}(z)-f(z)\right|{ }^{p} n(z)|d z| \leqq 2 \pi n^{\prime \prime}\left(M^{\prime} R^{n}\right)^{p},
$$

which implies the inequality

$$
\int_{C}\left|F_{n}(z)-f(z)\right|{ }^{p} n(z)|d z| \leqq 2 \pi n^{\prime \prime}\left(M^{\prime} R^{n}\right)^{p},
$$

for the functions $F_{n}(z)$ of best approximation, and this in turn implies

$$
\int_{C}\left|F_{n}(z)-f(z)\right| p|d z| \leqq 2 \pi \frac{n^{\prime \prime}}{n^{\prime}}\left(M^{\prime} R^{n}\right)^{p} .
$$

We shall have occasion to apply the following lemma:*

LEMMA II. If each of the functions $\phi_{n}(z)_{3} n=1,2, \cdots$, is analytic on and within the circle $C$, and if we set

$$
\int_{C}\left|\phi_{n}(z)\right| p|d z|=\epsilon_{n}, p>0,
$$

then we have for $z$ on an arbitrary closed point set $C^{\prime}$ interior to $C$

$$
\left|\phi_{n}(z)\right| \leqq Q \epsilon_{n}^{1 / p}
$$

where $Q$ depends on $C^{\prime}$ but not on $\phi_{n}(z)$.

If we restrict $z$ so that we have $|z| \leqq Z<1$, then we have by (8.5) and (8.6) for suitable choice of $M$ (independent of $n$ )

$$
\left|F_{n}(z)-f(z)\right| \leqq M R^{n} .
$$

By the use of (8.1) we find, for suitable $M_{1}$,

$$
\left|f_{n}(z)-F_{n}(z)\right| \leqq M_{1} R^{n},|z| \leqq Z<1,
$$

* Walsh, these Transactions, vol. 33 (1931), pp. 370-388. 
and by Lemma I we have

$$
\left|f_{n}(z)-F_{n}(z)\right| \leqq M_{1} R^{n}\left(\frac{A^{\prime} R_{1}-1}{A^{\prime}-R_{1}}\right)^{n}, \text { for }|z| \leqq Z R_{1}, 1<R_{1}<A^{\prime}<A
$$

Inequality (8.7) is valid for every $Z<1$ and for every $R>(T+A) /(1+A T)$ and for every $A^{\prime}<A$. It follows, precisely as in case (1), that the sequence $F_{n}(z)$ converges for

$$
|z|<\frac{A^{2} T+T+2 A}{2 A T+A^{2}+1} \text {, uniformly for }|z| \leqq R_{1}^{\prime}<\frac{A^{2} T+T+2 A}{2 A T+A^{2}+1},
$$

and Theorem IV is proved in case (2).

We take up now the remaining case (3) of Theorem IV. Let $F_{n}(z)$ now denote the rational function of degree $n$ with the prescribed poles of best approximation to $f(z)$ over the area $S:|z| \leqq 1$ in the sense of least weighted $p$ th powers, that is, the admissible function such that

$$
\iint_{S}\left|F_{n}(z)-f(z)\right|^{p} n(z) d S, \quad p>0,
$$

is not greater than the corresponding integral for any other admissible function. The function $n(z)$ is supposed positive and continuous on $S$, and under these conditions a function $F_{n}(z)$ of best approximation always exists, and is unique if $p>1$.

Denote by $n^{\prime}$ and $n^{\prime \prime}$ two numbers such that we have

$$
0<n^{\prime}<n(z)<n^{\prime \prime}, \quad z \text { on } S .
$$

For the functions $f_{n}(z)$ of Theorem I we have by (8.1)

$$
\iint_{S}\left|f_{n}(z)-f(z)\right|^{p} n(z) d S \leqq \pi n^{\prime \prime}\left(M^{\prime} R^{n}\right)^{p},
$$

which implies the inequality

$$
\iint_{S}\left|F_{n}(z)-f(z)\right|^{p} n(z) d S \leqq \pi n^{\prime \prime}\left(M^{\prime} R^{n}\right)^{p},
$$

for the functions $F_{n}(z)$ of best approximation, and this implies in turn

$$
\iint_{S}\left|F_{n}(z)-f(z)\right|^{p} d S \leqq \pi \frac{n^{\prime \prime}}{n^{\prime}}\left(M^{\prime} R^{n}\right)^{p}
$$

We are now ready to apply*

* Walsh, these Transactions, vol. 33 (1931), pp. 370-388. 
LEMMA III. Let $C$ be an arbitrary closed limited region. If each of the functions $\phi_{n}(z), n=1,2, \cdots$, is analytic in $C$, and if we set

$$
\iint_{C}\left|\phi_{n}(z)\right|^{p} d S=\epsilon_{n}, p>0
$$

then for $z$ on any closed point set $C^{\prime}$ interior to $C$ we have

$$
\left|\phi_{n}(z)\right|^{p} \leqq Q \epsilon_{n}^{1 / p},
$$

where $Q$ depends on $C^{\prime}$ but not on $\phi_{n}(z)$.

If we restrict $z,|z| \leqq Z<1$, the inequality

$$
\left|F_{n}(z)-f(z)\right| \leqq M R^{n},
$$

where $M$ depends on $Z$ but not on $n$, thus holds. The proof used in case (2) can now be followed directly, as the reader will verify, and this completes the proof of Theorem IV in all the cases mentioned.

In Theorem IV we have assumed the points $\alpha_{i n}$ to have no limit point interior to the circle $|z|=A>1$. It is naturally possible to use here an arbitrary circular region exterior to $|z| \leqq 1$ as the region to which the limit points of the set $\alpha_{\text {in }}$ are restricted, and even to prove still broader results. We state the general theorem involved. The results are completely analogous to the results of $\S 6$, and in fact include Theorem II as a special case. The proofs here are left to the reader; they depend particularly on the fact that under a linear transformation of the complex variable which transforms $|z| \leqq 1$ into itself, the integrals

$$
\int_{C}\left|f_{n}(z)-f(z)\right|{ }^{p} n(z)|d z|, \iint_{|z| \leqq 1}\left|f_{n}(z)-f(z)\right|^{p} n(z) d S, p>0,
$$

are transformed into integrals of the same type with new (positive and continuous) norm functions $n(z)$.

THEOREM IVa. If the numbers $\alpha_{\text {in }}$ have no limit point $z$ such that $|(\alpha z+\beta) /(\gamma z+\delta)|<A>1$, and if the function $f(z)$ is analytic for $\mid(\alpha z+\beta) /(\gamma z$ $+\delta) \mid\left\langle T>1\right.$, where $|\alpha / \gamma|>1, \alpha \delta-\beta \gamma \neq 0$, then the sequence $\left\{F_{n}(z)\right\}$ of rational functions of respective degrees $n$ with poles $\alpha_{i n}$ of best approximation to $f(z)$ on $C:|(\alpha z+\beta) /(\gamma z+\delta)|=1$ converges to the limit $f(z)$ for

$$
\left|\frac{\alpha z+\beta}{\gamma z+\delta}\right|<\frac{A^{2} T+T+2 A}{2 A T+A^{2}+1} \text {, uniformly for }\left|\frac{\alpha z+\beta}{\gamma z+\delta}\right| \leqq R<\frac{A^{2} T+T+2 A}{2 A T+A^{2}+1} \text {. }
$$

The measures of approximation contemplated here are naturally (1), (2), (3), and the restriction $|\alpha / \gamma|>1$ is made simply to avoid an improper in- 
tegral if (3) is the measure of approximation, taken over $\mid(\alpha z+\beta) /(\gamma z$ $+\delta) \mid \leqq 1$. If (1) or (2) is used, this restriction may be omitted, and it may even be omitted in case (3) if suitably modified restrictions on the norm function are imposed for the case that $|(\alpha z+\beta) /(\gamma z+\delta)| \leqq 1$ is an infinite region.

It will be noticed that throughout $\$ 8$ we have not explicitly used the fact that we were dealing with sequences of rational functions of best approximation. It is in every case sufficient if we have sequences which converge (as measured by any one of the several measures of approximation) like the sequence of best approximation, in the sense of geometric inequalities as used.

We remark that inequality (8.1), holding for $z$ on $C$ for an arbitrary $R>(T+A) /(1+A T)$, cannot be proved to hold, for an arbitrary choice of the $\alpha_{\text {in }}$ and for an arbitrary function $f(z)$ analytic for $|z|<T$, for an arbitrary $R>R^{\prime}<(T+A) /(1+A T)$. This follows from the specific example given in \$5. From (5.1) we have for $z$ on $C$

$$
\left|f(z)-f_{n}(z)\right|=\frac{1}{T|z+T|}\left(\frac{T+A}{1+A T}\right)^{n} .
$$

Overconvergence, in the form in which we have proved it in cases (1), (2), (3), and so far as the present methods are concerned, is not a consequence of the inequalities (8.2), (8.5), (8.8) alone, but is a consequence of those inequalities together with our knowledge of the-fact that $f(z)$ is analytic for $|z|<T$. If we know merely that for some function $f(z)$ defined for $|z| \leqq 1$, one of the inequalities (8.2), (8.5), (8.8) holds, then we can prove only that the sequence $f_{n}(z)$ converges and that $f(z)$ is analytic for $|z|<\left(A+R^{1 / 2}\right) /\left(1+A R^{1 / 2}\right)$. We have from (8.2), for instance,

$$
\left|F_{n}(z)-F_{n+1}(z)\right| \leqq M^{\prime}(1+R) R^{n}, z \text { on } C
$$

where $R$ is an arbitrary number less than unity. By Lemma $I$ this yiel 1 since the function on the left is a rational function of degree $2 n+1$,

$$
\left|F_{n}(z)-F_{n+1}(z)\right| \leqq M^{\prime}(1+R) R^{n}\left(\frac{A^{\prime} R_{1}-1}{A^{\prime}-R_{1}}\right)^{2 n+1}, \quad|z|=R_{1}>1, A^{\prime}<A .
$$

Thus the sequence $F_{n}(z)$ converges for $R_{1}<\left(A+R^{1 / 2}\right) /\left(1+A R^{1 / 2}\right)$, that is, for $|z|<\left(A+R^{1 / 2}\right) /\left(1+A R^{1 / 2}\right)$, uniformly for $|z| \leqq Z<\left(A+R^{1 / 2}\right) /\left(1+A R^{1 / 2}\right)$.

9. Interpolation at the origin. As a matter of interest, we study the rational functions (2.1) of respective degrees $n$ with poles in the prescribed points $\alpha_{i n}$ which are defined from the given analytic function $f(z)$ by interpolation in the origin, and we shall investigate the convergence of the sequence so determined. That is to say, the present functions $f_{n}(z)$ shall have the property 


$$
f_{n}^{(k)}(0)=f^{(k)}(0) \quad(k=0,1, \cdots, n),
$$

where $f(z)$ is the given function analytic for $|z|<T$. The conditions (9.1) determine $f_{n}(z)$. completely. Under the present circumstances we have the formula

(9.2) $f(z)-f_{n}(z)=\frac{1}{2 \pi i} \int_{C^{\prime}} f(t) \frac{z^{n+1}\left(t-\alpha_{1}\right)\left(t-\alpha_{2}\right) \cdots\left(t-\alpha_{n}\right)}{\left(z-\alpha_{1}\right)\left(z-\alpha_{2}\right) \cdots\left(z-\alpha_{n}\right)(t-z) t^{n+1}} d t ;$

let us verify the correctness of this formula. The function

$$
f_{n}(z)=\frac{1}{2 \pi i} \int_{C^{\prime}}\left[1-\frac{z^{n+1}\left(t-\alpha_{1}\right)\left(t-\alpha_{2}\right) \cdots\left(t-\alpha_{n}\right)}{\left(z-\alpha_{1}\right)\left(z-\alpha_{2}\right) \cdots\left(z-\alpha_{n}\right) t^{n+1}}\right] f(t) \frac{d t}{t-z}
$$

is a rational function of degree $n$; indeed, if the quantities in the square bracket are reduced to a common denominator and added, the new numerator considered as a function of $z$ and $t$ vanishes for $t=z$ and hence is divisible by $t-z$. The function $f_{n}(z)$ defined by (9.3) is of degree $n$ and has (formally) the prescribed poles. Moreover, the right-hand member of (9.2) vanishes together with its first $n$ derivatives for $z=0$, so the verification is complete. We shall prove

THEOREM Va. Let the function $f(z)$ be analytic for $|z|<T$ and let the numbers $\alpha_{i n}, i=1,2, \cdots, n ; n=1,2, \cdots$, be preassigned and have no limit point whose modulus is less than $A$. Denote by $f_{n}(z)$ the rational function of the form (2.1) which satisfies (9.1). Then the sequence $\left\{f_{n}(z)\right\}$ approaches the limit $f(z)$ for $|z|<A T /(A+2 T)$, uniformly for $|z|<R<A T /(A+2 T)$.

For $|t|=T^{\prime}<T$ and $|z|=Z<A^{\prime}$, we have for $|\alpha| \geqq A^{\prime}<A$

$$
\left|\frac{t-\alpha}{z-\alpha}\right| \leqq \frac{T^{\prime}+A^{\prime}}{A^{\prime}-Z}
$$

Under the same restrictions we have

$$
\left|\frac{z\left(t-\alpha_{k}\right)}{t\left(z-\alpha_{k}\right)}\right| \leqq \frac{Z\left(T^{\prime}+A^{\prime}\right)}{T^{\prime}\left(A^{\prime}-Z\right)},
$$

so if we integrate over $C^{\prime}:|t|=T^{\prime}$, the right-hand member of (9.2) approaches zero provided we have

$$
\frac{Z\left(T^{\prime}+A^{\prime}\right)}{T^{\prime}\left(A^{\prime}-Z\right)}<1 \text {, or } Z<\frac{A^{\prime} T^{\prime}}{A^{\prime}+2 T^{\prime}} .
$$

Since $T^{\prime}<T$ and $A^{\prime}<A$ are arbitrary, we have convergence for $Z<A T /(A$ $+2 T)$, uniform convergence for $Z<R<A T /(A+2 T)$. In particular if $A=T$, 
we have convergence for $|z|<A / 3$. If the function $f(z)$ is analytic at every finite point of the plane, we may allow $T^{\prime}$ to become infinite; the quantity $A T /(A+2 T)$ approaches $A / 2$. If we have $\lim _{n \rightarrow \infty} \alpha_{i n}=\infty$ uniformly, then we may allow $A$ to become infinite; the limit of $A T /(A+2 T)$ is $T$.

The significance of the case that $A$ is infinite deserves further discussion. Throughout our work on interpolation as well as on approximation any or all of the points $\alpha_{i n}$ may naturally be the point at infinity. If the points $\alpha_{i n}$ have no limit point except at infinity, then the sequence $f_{n}(z)$ converges to $f(z)$ uniformly for $|z| \leqq Z<T$. The case $\alpha_{i n}=\infty$ is included here, which corresponds of course to the expansion of $f(z)$ in Taylor's series. Theorem Va, like most of the other results of the present paper, thus deals with a generalization of Taylor's series.

The limit $A T /(A+2 T)$ that we have derived can be replaced by no larger limit, as we now point out. Take $f(z)=1 /(z+T)$, so that we have for $\alpha_{i n}=A$, $f_{n}(z)=\frac{T^{n+1}(z-A)^{n}+(T+A)^{n} z^{n+1}}{T^{n+1}(z+T)(z-A)^{n}}, f_{n}(z)-f(z)=\frac{(T+A)^{n} z^{n+1}}{T^{n+1}(z+T)(z-A)^{n}} ;$

the reader can readily verify these formulas. The equation

$$
\lim _{n \rightarrow \infty} f_{n}(z)=f(z)
$$

is valid whenever $|(T+A) z /(T(z-A))|<1$, and cannot hold if we have $|(T+A) z /(T(z-A))|>1$. Indeed, we have divergence of the sequence $f_{n}(x)$ if $|z /(z-A)|>T /(T+A)$, and in particular we have divergence for $z=A T /(A+2 T)$.

10. Interpolation at origin; continuation. In the present section we continue the study of the sequence $f_{n}(z)$ found by interpolation in the origin, where now the limit points of the numbers $\alpha_{i n}$ are restricted to lie in some circular region $\Gamma$ bounded by a circle whose center is not necessarily the origin.

A sufficient condition for the equation $\lim _{n \rightarrow \infty} f_{n}(z)=f(z)$ is still

$$
\left|\frac{z}{z-\alpha}\right|<\left|\frac{t}{t-\alpha}\right|
$$

for the numbers $\alpha$ involved, so we study this inequality in more detail. If the region $\Gamma$ is comparatively small, and does not contain the origin, the locus of points $z$ such that

$$
\left|\frac{z}{z-\alpha}\right|=k
$$


where $k$ is a given positive constant and $\alpha$ takes on all possible values in the region $\Gamma$, is a portion of a plane bounded by a certain Cartesian oval.* The Cartesian oval consists of two non-intersecting curves $C_{1}$ and $C_{2}$, each of which separates the origin and the region $\Gamma$. Let $C_{1}$ separate $O$ and $C_{2}$. Denote by $\Gamma_{1}$ and $\Gamma_{2}$ the two open regions bounded by $C_{1}$ and $C_{2}$ respectively, which are mutually exclusive and contain $O$ and $\Gamma$ respectively. The locus of points $z$ such that (10.2) is valid is the region between and bounded by $C_{1}$ and $C_{2}$. Thus for all points $z$ of $\Gamma_{1}$ we have

$$
\left|\frac{z}{z-\alpha}\right|<k,
$$

no matter what the choice of $\alpha$ in $\Gamma$ may be, and for all points $t$ of $\Gamma_{2}$ we have

$$
\left|\frac{t}{t-\alpha}\right|>k
$$

no matter what the choice of $\alpha$ in $\Gamma$ may be. The proofs of (10.3) and (10.4) are practically obvious; for instance (10.3) is valid for $z=0$ no matter what $\alpha$ in $\Gamma$ may be, and hence is valid for every other value of $z$ of $\Gamma_{1}$.

If the given function $f(z)$ is analytic in the closed region $\Gamma_{2}^{\prime}$ complementary to $\Gamma_{2}$, we may take the integral in (9.2) over a path interior to $\Gamma_{2}, \dagger$ so that (10.1) is satisfied for all points $z$ of $\Gamma_{1}$. We have

$$
\lim _{n \rightarrow \infty} f_{n}(z)=f(z)
$$

uniformly for $z$ in $\Gamma_{1}$. If $\Gamma_{2}^{\prime}$ is the largest region of the kind described (that is, we use the largest possible value of $k$ ) within which $f(z)$ is analytic, we have $\lim _{n \rightarrow \infty}$ $f_{n}(z)=f(z)$ for $z$ interior to the corresponding $\Gamma_{1}$, uniformly for $z$ on any closed point set interior to $\Gamma_{1}$. This condition is the precise analogue of the condition found in $\$ 9$. Indeed, that previous condition is included under the present one.

The condition just considered has been established only under the assumption that the given region $\Gamma$ is sufficiently small. Let $\Gamma$ be now any circular region not containing the origin. If $k$ starts at zero and becomes larger, the curve $C_{2}$ sweeps out the entire plane and eventually reduces to a point, later, for still larger values of $k$, expanding again. But for these larger values of $k$ the curve $C_{2}$ no longer has any significance in the study of the locus (10.2);

* Walsh, Quarterly Journal of Mathematics, vol. 50 (1924), pp. 154-165.

$\dagger$ This integration is valid even if the region $\Gamma_{2}{ }^{\prime}$ contains in its interior the point at infinity, for under such circumstances the function $f(t)$ is analytic at infinity and the integrand in (9.2) has a zero of order at least two at $t=\infty$. 
the locus (10.2) is bounded by the single oval $C_{1}$. The condition we have developed above is valid, no matter what may be the circular region $\Gamma$ not containing the origin, provided that the locus (10.2) is bounded by two nonintersecting curves $C_{1}$ and $C_{2}$; the value of $k$ is thus uniquely determined. In particular there is a limiting case in which $C_{2}$ reduces to a point, and the only singularity of $f(z)$ lies at this point. The discussion is valid essentially as given, also in this limiting case.

Another method of studying convergence in the case that the $\alpha_{i n}$ have no limit point exterior to a circular region $\Gamma$ not containing $O$, is to transform by means of a linear transformation of the form $w=z /(z-\beta)$, where $\beta$ is the inverse of $O$ in the circle $\Gamma$. The origin $z=0$ is transformed into the origin $w=0$, and the circle $\Gamma$ is transformed into a circle whose center is the origin. The region $\Gamma$ is transformed into the exterior of this new circle. A rational function $f_{n}(z)$ of degree $n$ with poles in the points $\alpha_{i n}$ which satisfies the equations

$$
f_{n}^{(k)}(0)=f^{(k)}(0) \quad(k=0,1,2, \cdots, n)
$$

is transformed into a rational function $f_{n}(\beta w /(w-1))$ of $w$ of degree $n$ with poles in the transforms of the points $\alpha_{i n}$ which satisfies the equations

$$
\frac{d^{k}}{d w^{k}} f_{n}\left(\frac{\beta w}{w-1}\right)=\frac{d^{k}}{d w^{k}} f\left(\frac{\beta w}{w-1}\right) \quad(k=0,1,2, \cdots, n),
$$

for the particular value $w=0$. The latter situation is treated in detail in $\$ 9$, so we may read off directly the results in the original situation in the $z$-plane.

If $f(z)$ is analytic for $|z /(z-\beta)|<T$ and if the numbers $\alpha_{i n}$ have no limit point $z$ such that $|z /(z-\beta)|<A$, then the sequence $f_{n}(z)$ approaches $f(z)$ for $|z /(z-\beta)|<A T /(A+2 T)$, uniformly for $|z /(z-\beta)|<R<A T /(A+2 T)$. In particular if $A=T$, we have convergence for $|z /(z-\beta)|<A / 3$, uniform convergence for $|z /(z-\beta)|<R<A / 3$. If $f(z)$ is analytic at every point of the plane except the point $z=\beta$, we have $\lim _{n \rightarrow \infty} f_{n}(z)=f(z)$ for $|z /(z-\beta)|<A / 2$, uniformly for $|z /(z-\beta)|<R<A / 2$. If the only limit point of the $\alpha_{i n}$ is the point $\beta, f(z)$ analytic for $|z /(z-\beta)|<T$, we have this equation for $|z /(z-\beta)|$ $<T$, uniformly for $|z /(z-\beta)|<R<T$.

11. Interpolation at the roots of unity. Another method of approximating to a given analytic function by rational functions of the form (2.1) is suggested by well known work of Runge* and Fejér $\dagger$ on approximation by polynomials and consists in interpolating in the roots of unity. Let us establish

* Theorie und Praxis der Reihen, Leipzig, 1904, pp. 126-142.

† Göttinger Nachrichten, 1918, pp. 319-331. 
TheOREM VI. Let the function $f(z)$ be analytic for $|z|<T>1$ and let the numbers $\alpha_{i n}, i=1,2, \cdots, n ; n=1,2, \cdots$, be preassigned and have no limit point whose modulus is less than $A>1$. Denote by $f_{n}(z)$ the rational function of the form (2.1) which coincides with $f(z)$ in the $(n+1)$ st roots of unity. Then the sequence $\left\{f_{n}(z)\right\}$ approaches the limit $f(z)$ for $|z|<A T /(A+2 T)$, uniformly for $|z|<R<A T /(A+2 T)$ when $A T /(A+2 T)>1$.

Let us write down the formula for $f_{n}(z)$ and then verify it. We have

$$
\begin{aligned}
& f(z)-f_{n}(z)=\frac{1}{2 \pi i} \int_{C^{\prime}} f(t) \frac{\left(z^{n+1}-1\right)\left(t-\alpha_{1}\right)\left(t-\alpha_{2}\right) \cdots\left(t-\alpha_{n}\right)}{\left(z-\alpha_{1}\right)\left(z-\alpha_{2}\right) \cdots\left(z-\alpha_{n}\right)(t-z)\left(t^{n+1}-1\right)} d t \\
& f_{n}(z)=\frac{1}{2 \pi i} \int_{C^{\prime}} f(t)\left[1-\frac{\left(z^{n+1}-1\right)\left(t-\alpha_{1}\right)\left(t-\alpha_{2}\right) \cdots\left(t-\alpha_{n}\right)}{\left(z-\alpha_{1}\right)\left(z-\alpha_{2}\right) \cdots\left(z-\alpha_{n}\right)(t-z)\left(t^{n+1}-1\right)}\right] d t
\end{aligned}
$$

These integrals are to be taken over a circle $C^{\prime}:|t|=T^{\prime}<T, T^{\prime}>1$. Indeed, if we reduce the two quantities in the square bracket in (11.2) to a common denominator, the factor $t-z$ cancels from numerator and denominator, for the numerator vanishes (considered as a function of $t$ and $z$ ) for $t=z$. Thus (11.2) defines $f_{n}(z)$ as a rational function of $z$ of degree $n$ with the proper denominator. It appears from (11.1) that the function $f_{n}(z)$ as so defined coincides with $f(z)$ in the points $z$ for which $z^{n+1}=1$; these properties define $f_{n}(z)$ uniquely.

The integral in (11.1) approaches zero with $1 / n$ for $|z|=Z \geqq 1, Z<A^{\prime}$ provided we have

$$
\frac{Z\left(T^{\prime}+A^{\prime}\right)}{\left(A^{\prime}-Z\right) T^{\prime}}<1
$$

where $A^{\prime}$ is an arbitrary number less than $A$. Condition (11.3) is equivalent to $Z<A^{\prime} T^{\prime} /\left(A^{\prime}+2 T^{\prime}\right)$. Thus, by the arbitrariness of $A^{\prime}<A$ and $T^{\prime}<T$, if $A T /(A+2 T)>1$, we have

(11.4) $\lim _{n \rightarrow \infty} f_{n}(z)=f(z)$ for $|z|<\frac{A T}{A+2 T}$, uniformly for $|z| \leqq R<\frac{A T}{A+2 T}$.

If we do not have $A T /(A+2 T)>1$, we can still study convergence interior to the unit circle. The integral in (11.1) approaches zero with $1 / n$, for $|z| \leqq Z \leqq 1$, provided we have $\left(T^{\prime}+A^{\prime}\right) /\left(\left(A^{\prime}-Z\right) T^{\prime}\right)<1$, that is, provided we have $Z<\left(A^{\prime} T^{\prime}-T^{\prime}-A^{\prime}\right) / T^{\prime}$. Again we use the arbitrariness of $A^{\prime}<A$ and of $T^{\prime}<T$; it follows that we have

$$
\begin{aligned}
& \lim _{n \rightarrow \infty} f_{n}(z)=f(z) \text { for }|z|<(A T-A-T) / T \\
& \quad \text { uniformly for }|z| \leqq R<(A T-A-T) / T
\end{aligned}
$$


when $(A T-A-T) / T \leqq 1$, and uniformly for $|z| \leqq 1$ when $(A T-A-T) / T$ $>1$. It will be noticed that the quantity $(A T-A-T) / T$ may be negative or zero, in which case we draw no conclusion regarding convergence for $|z| \leqq 1$. The two conditions $A T /(A+2 T)>1$ and $(A T-A-T) / T>1$ are the same. If this condition is satisfied, we use (11.4), and if this condition is not satisfied we use (11.5).

Let us choose a specific example to show that the limits obtained in (11.4) and (11.5) are not artificial, but cannot be replaced (for the general function $f(z)$ and for arbitrary $\left.\alpha_{i n}\right)$ by any larger limits. We set $f(z)=1 /(z+T), T>1$, $\alpha_{i n}=A>1$, and it is a simple matter of verification to show that the function $f_{n}(z)$ yields

$$
\begin{aligned}
f_{n}(z)-f(z) & =\frac{(T+A)^{n}\left(z^{n+1}-1\right)}{\left[(-1)^{n}+T^{n+1}\right](z+T)(z-A)^{n}} \\
& =\left(\frac{T+A}{T} \frac{z}{z-A}\right)^{n} \frac{1-\frac{1}{z^{n+1}}}{\left[\frac{(-1)^{n}}{T^{n}}+T\right](z+T)}
\end{aligned}
$$

A necessary and sufficient condition for the approach of this quantity to zero with $1 / n$, in the case $|z|>1$, is

$$
\frac{T+A}{T}\left|\frac{z}{z-A}\right|<1
$$

and this condition fails even for $z=A T /(A+2 T)$. For $|z|<1$, we can write (11.6) in the form

$$
f_{n}(z)-f(z)=\left[\frac{T+A}{T(z-A)}\right]^{n} \frac{1-z^{n+1}}{\left[\frac{(-1)^{n}}{T^{n}}+T\right](z+T)},
$$

and a necessary and sufficient condition for the approach of this quantity to zero with $1 / n$ is

$$
\frac{T+A}{T|z-A|}<1
$$

This condition fails even for $z=(A T-A-T) / T$.

Condition (11.4) is just the condition found in $\$ 9$, so we refer to that place for a discussion of the cases $A=T, A=\infty, T=\infty$. Moreover, the condition 


$$
\left|\frac{z}{z-\alpha}\right|<\left|\frac{t}{t-\alpha}\right|
$$

is studied in some detail in $\$ 10$, where the numbers $\alpha$ lie or more generally have no limit point in a circular region not necessarily concentric with the origin, and this yields results in the present case on convergence of the sequence $\left\{f_{n}(z)\right\}$, provided $|z|>1$.

If the points $\alpha_{i n}$ have no limit point exterior to the circular region $\Gamma$ (exterior to $C$ ), our condition for convergence for $|z|<1$ is

$$
\left|\frac{t-\alpha}{t}\right|<|z-\alpha| .
$$

Again the path of integration $C^{\prime}$ in (11.2) can be chosen so that on it we have

$$
\left|\frac{t}{t-\alpha}\right|>k,
$$

where this inequality holds uniformly for all $t$ on the path of integration and for all $\alpha$ in $\Gamma$, and provided that $f(z)$ is analytic in the closed region bounded by $C^{\prime}$ and containing $C:|z|=1$. Ther we have $\lim _{n \rightarrow \infty} f_{n}(z)=f(z)$ uniformly for $|z-\alpha| \geqq 1 / k$. This latter condition is the requirement that $z$ should lie in a certain infinite circular region concentric with $\Gamma$, and is naturally to be taken in conjunction with $|z|<1$.

It is instructive to compare the difference $f(z)-f_{n}^{0}(z)$ for interpolation in the origin with the difference $f(z)-f_{n}(z)$ for interpolation in the $(n+1)$ st roots of unity. The difference between these two differences is

$$
\begin{aligned}
& f_{n}^{0}(z)-f_{n}(z) \\
& =\frac{1}{2 \pi i} \int f(t) \frac{\left(t-\alpha_{1}\right)\left(t-\alpha_{2}\right) \cdot \cdot\left(t-\alpha_{n}\right) d t}{\left(z-\alpha_{1}\right)\left(z-\alpha_{2}\right) \cdots\left(z-\alpha_{n}\right)(t-z)}\left[\frac{z^{n+1}}{t^{n+1}}-\frac{z^{n+1}-1}{t^{n+1}-1}\right]
\end{aligned}
$$

the square bracket reduces to

$$
\frac{t^{n+1}-z^{n+1}}{t^{n+1}\left(t^{n+1}-1\right)}
$$

The integral is to be taken over a circle whose radius $T^{\prime}$ is greater than unity, for otherwise we cannot be sure of interpolation in the $(n+1)$ st roots of unity. We consider $|z|=Z<A^{\prime}<A$. There are two cases according as $Z \leqq T^{\prime}<T$ or $Z>T^{\prime}$; the integrand in (11.7) has no singularity for $t=z$, so the equation is valid even if $Z>T$.

A sufficient condition for the convergence to zero of the integral in (11.7) is in these respective cases 


$$
\begin{aligned}
& \left|\frac{t-\alpha_{k}}{t} \frac{1}{z-\alpha_{k}}\right|<P<1, \frac{T^{\prime}+A^{\prime}}{T^{\prime}} \frac{1}{A^{\prime}-Z}<1, Z<\frac{A^{\prime} T^{\prime}-T^{\prime}-A^{\prime}}{T^{\prime}} \\
& \left|\frac{t-\alpha_{k}}{t^{2}} \frac{z}{z-\alpha_{k}}\right|<P<1, \frac{T^{\prime}+A^{\prime}}{A^{\prime}-Z} \frac{Z}{T^{\prime 2}}<1, \quad Z<\frac{A^{\prime} T^{\prime 2}}{T^{\prime 2}+T^{\prime}+A^{\prime}}
\end{aligned}
$$

The conditions $(A T-T-A) / T>T, A T^{2} /\left(T^{2}+T+A\right)>T$, and $(A T-T$ $-A) / T>A T^{2} /\left(T^{2}+T+A\right)$ are precisely the same. Thus $f_{n}^{0}(z)-f_{n}(z)$ approaches zero for $|z|<(A T-T-A) / T$, uniformly for $|z|<R<(A T-T$ $-A) / T$, provided that this last quantity is less than or equal to $T$, and $f_{n}^{0}(z)-f_{n}(z)$ approaches zero for $|z|<A T^{2} /\left(T^{2}+T+A\right)$, uniformly for $|z|<R<A T^{2} /\left(T^{2}+T+A\right)$ provided this last expression is greater than $T$. Thus we may have $\lim _{n \rightarrow \infty}\left[f_{n}^{0}(z)-f_{n}(z)\right]=0$ uniformly in a region which contains a singularity of $f(z)$, and in any case this limit that we have found if greater than unity is greater than the common limit found in the two cases, for the conditions $\lim _{n \rightarrow \infty} f_{n}^{0}(z)=f(z), \lim _{n \rightarrow \infty} f_{n}(z)=f(z)$. Indeed, the condition $(A T-T$ $-A) / T>A T /(A+2 T)$ is equivalent to the condition $(A+T)(A T-A-2 T)$ $>0$, which is precisely the condition that $(A T-T-A) / T$ or $A T /(A+2 T)$ should be greater than unity.

It is instructive to verify the fact just proved, for the particular function $f(z)=1 /(z+T)$.

There are cases where the points of interpolation are on $|z|=1$ but not exactly the points $1^{1 /(n+1)}$, where the results are likewise comparable to those for interpolation in the origin. It would be of interest to determine precise geometric conditions that this be true; algebraic conditions are readily obtainable from the formulas we have used.

12. Interpolation in arbitrary points. We sketch rapidly some results which include the main results of $\$ 9$ as a special case, and which also throw some light on the results of $\$ 11$. We consider the rational function $f_{n}(z)$ of form (2.1) which takes on the values of the given function $f(z)$ in $n+1$ arbitrary points $\beta_{1 n}, \beta_{2 n}, \cdots, \beta_{n+1, n}$ (the second subscript will frequently be dropped for simplicity) and shall determine sufficient conditions for the approach of $f_{n}(z)$ to $f(z)$ as $n$ becomes infinite.

THEOREM V. Let the function $f(z)$ be analytic for $|z|<T$ and let the numbers $\alpha_{\text {in }}$ have no limit point whose modulus is less than $A$, and the numbers $\beta_{\text {in }}$ no limit point whose modulus is greater than $B<A, B<T$. Then the sequence of rational functions $f_{n}(z)$ of respective degrees $n$ with poles in the points $\alpha_{\text {in }}$ which coincide with the values of $f(z)$ in the points $\beta_{i n}$, approaches the limit $f(z)$ for $|z|<(A T-B T-2 A B) /(A-B+2 T)$, uniformly for $|z|<R<(A T-B T$ $-2 A B) /(A-B+2 T)$, provided $A T-B T-2 A B>0$. 
The rational function $f_{n}(z)$ is completely determined by the prescribed conditions. The reader will verify the formula

(12.1) $f(z)-f_{n}(z)$

$=\frac{1}{2 \pi i} \int_{C^{\prime}} f(t) \frac{\left(z-\beta_{1}\right)\left(z-\beta_{2}\right) \cdots\left(z-\beta_{n+1}\right)\left(t-\alpha_{1}\right)\left(t-\alpha_{2}\right) \cdots\left(t-\alpha_{n}\right)}{\left(z-\alpha_{1}\right)\left(z-\alpha_{2}\right) \cdots\left(z-\alpha_{n}\right)(t-z)\left(t-\beta_{1}\right)\left(t-\beta_{2}\right) \cdots\left(t-\beta_{n+1}\right)} d t$,

where we take the integral over a circle $|t|=T^{\prime}<T, T^{\prime}>B^{\prime}>B$. We consider moreover $|z|=Z<A$ to be fixed during the integration and choose an arbitrary $A^{\prime}<A$. The right-hand member of (12.1) approaches zero uniformly in $z$ as $n$ becomes infinite provided we have for $n$ sufficiently large

$$
\left|\frac{t-\alpha_{i}}{z-\alpha_{i}} \frac{z-\beta_{i}}{t-\beta_{i}}\right|<1,
$$

hence provided we have

$$
\begin{gathered}
\frac{T^{\prime}+A^{\prime}}{A^{\prime}-Z} \frac{Z+B^{\prime}}{T^{\prime}-B^{\prime}}<1, \\
Z<\frac{A^{\prime} T^{\prime}-B^{\prime} T^{\prime}-2 A^{\prime} B^{\prime}}{A^{\prime}-B^{\prime}+2 T^{\prime}} .
\end{gathered}
$$

To be sure, in evaluating the right-hand member of (12.1), the second factor in the left-hand member of (12.2) is to be considered raised to the power $n+1$, and the first factor only to the power $n$. But by (12.3). itself, the second factor is uniformly limited in absolute value, so in (12.2) we may take both factors to the power $n$.

When we remember that $A^{\prime}<A, B^{\prime}>B, T^{\prime}<T$ are arbitrary, we see from (12.4) that the proof of Theorem $V$ is complete. If all the points $\beta_{i}$ are not distinct, Theorem $\mathrm{V}$ is interpreted to mean the coincidence of various derivatives of $f_{n}(z)$ with those of $f(z)$ at multiple points $\beta_{i}$, and this condition is fulfilled in (12.1). If $B=0$, we have the situation of Theorem Va. If $A=\infty$ (of which interpolation by polynomials is a special case), the limit in Theorem $\mathrm{V}$ is to be taken as $T-2 B$; if $T=\infty$ the limit is to be taken as $(A-B) / 2$; and if both $A$ and $T$ are infinite, this limit is to be taken as infinity. In particular, the inequality

$$
\frac{A T-B T-2 A B}{A-B+2 T}>B,
$$

which allows the points $\beta_{i}$ to be chosen arbitrarily with no limit point exterior to $|z|=B$ and assures uniform convergence of $f_{n}(z)$ to the function $f(z)$ for $|z|=B$, is equivalent to $T>\left(3 A B-B^{2}\right) /(A-3 B)$, provided $A>3 B$. 
The general condition for convergence

$$
|z|<\frac{A T-B T-2 A B}{A-B+2 T}
$$

of Theorem $\mathrm{V}$ can be replaced by no condition

$$
|z|<R>\frac{A T-B T-2 A B}{A-B+2 T}
$$

as we now show by an example. Take $f(z)=1 /(z+T), \beta_{i}=-B, \alpha_{i}=A$. Then we find

$$
\begin{aligned}
f_{n}(z)-f(z) & =\frac{(T+A)^{n}(z+B)^{n+1}}{(T-B)^{n+1}(z+T)(z-A)^{n}} \\
& =\left(\frac{T+A}{T-B} \frac{z+B}{z-A}\right)^{n} \frac{z+B}{(T-B)(z+T)}
\end{aligned}
$$

For the value $z=(A T-B T-2 A B) /(A-B+2 T)$ we have

$$
\frac{T+A}{T-B} \frac{z+B}{z-A}=-1
$$

so the last member of (12.5) diverges as $n$ becomes infinite.

If the numbers $\alpha_{\text {in }}$ and $\beta_{\text {in }}$ of Theorem $V$ are independent of $n$, the function $f_{n}(z)$ is the sum of the first $n+1$ terms of a series of the form

$$
a_{0}+a_{1} \frac{z-\beta_{1}}{z-\alpha_{1}}+a_{2} \frac{\left(z-\beta_{1}\right)\left(z-\beta_{2}\right)}{\left(z-\alpha_{1}\right)\left(z-\alpha_{2}\right)}+a_{3} \frac{\left(z-\beta_{1}\right)\left(z-\beta_{2}\right)\left(z-\beta_{3}\right)}{\left(z-\alpha_{1}\right)\left(z-\alpha_{2}\right)\left(z-\alpha_{3}\right)}+\cdots,
$$

where the given points $\alpha_{i}$ are supposed distinct from the point at infinity. If all of the $\alpha_{i}$ coincide with the point at infinity, the function $f_{n}(z)$ is the sum of the first $n+1$ terms of a series of the form

$$
a_{0}+a_{1}\left(z-\beta_{1}\right)+a_{2}\left(z-\beta_{1}\right)\left(z-\beta_{2}\right)+a_{3}\left(z-\beta_{1}\right)\left(z-\beta_{2}\right)\left(z-\beta_{3}\right)+\cdots
$$

These two types of series have been widely studied. The special case here that $\lim _{n \rightarrow \infty} \alpha_{n}$ and $\lim _{n \rightarrow \infty} \beta_{n}$ exist has recently been considered by Angelescu.*

Theorem $\mathrm{V}$ can be generalized as was Theorem Va, first making assumptions on the $\alpha_{i}, \beta_{i}$, and on the analyticity of $f(z)$ in certain circular regions bounded by circles of a coaxial family, then transforming these circles into a family of concentric circles.

\footnotetext{
* Bulletin, Académie Roumaine, vol. 9 (1925), pp. 164-168.-
} 
Let the function $f(z)$ be analytic for $|(z-a) /(z-b)|<T$ and let the numbers $\alpha_{i n}$ have no limit point $z$ such that $|(z-a) /(z-b)|<A$ and the numbers $\beta_{i n}$ no limit point $z$ such that $|(z-a) /(z-b)|>B<A, T$. Then the sequence of rational functions $f_{n}(z)$ of respective degrees $n$ with poles in the points $\alpha_{\text {in }}$ which coincide with the values of $f(z)$ in the points $\beta_{i n}$, approaches the limit $f(z)$ for

$\left|\frac{z-a}{z-b}\right|<\frac{A T-B T-2 A B}{A-B+2 T}$, uniformly for $\left|\frac{z-a}{z-b}\right|<R<\frac{A T-B T-2 A B}{A-B+2 T}$,

provided $A T-B T-2 A B>0$.

It will be noticed that formula (12.1) is valid even for an infinite region bounded by the curve $C^{\prime}$, provided that the points $\beta_{i}$ lie interior to this region and that $f(z)$ is analytic in the closed region. This follows from the fact that if $f(z)$ is analytic at infinity, the integrand of (12.1) has a zero for $t=\infty$ of at least the second order in $t$. The theorem just proved is naturally valid even if any of the regions $|(z-a) /(z-b)|<A, T,|(z-a) /(z-b)|>B$ is the exterior of a circle or even a half-plane.

The entire problem of studying the rational function $f_{n}(z)$ of degree $n$ with preassigned poles $\alpha_{i n}$ which coincides in $n+1$ preassigned points $\beta_{\text {in }}$ with a given analytic function $f(z)$ is invariant under linear transformation of the complex variable. That is to say, if all the points in the $z$-plane are transformed by a linear transformation, the function $f_{n}(z)$ is transformed into a rational function of degree $n$ whose poles lie in the transforms of the points $\alpha_{i n}$ and which coincides in the transforms of the points $\beta_{i n}$ with the analytic function which is the transform of the function $f(z)$. We shall now formulate the problem of the study of the convergence of the sequence $f_{n}(z)$ to the function $f(z)$ in a way more general than that previously done; the new formulation is expressed in terms of cross ratios and brings out clearly this invariant character.

Let $R_{1}$ and $R_{2}$ be arbitrary closed regions with no point in common. If the points $\alpha_{i n}$ lie in $R_{1}$ and the points $\beta_{i n}$ in $R_{2}$, what can be said of the convergence to the function $f(z)$ analytic in $R_{2}$ of the sequence of rational functions $f_{n}(z)$ of respective degrees $n$, whose poles lie in the prescribed points $\alpha_{i n}$, determined by interpolation in the points $\beta_{i n}$ ?

If there exists a curve $C^{\prime}$ bounding a region which contains $R_{2}$ in its interior but contains on or within it no singularity of $f(z)$, such that we have for every $t$ on $C^{\prime}$ and for every $z$ on $R_{2}$

$$
\left|\left(t, \alpha_{i n}, z, \beta_{i n}\right)\right|=\left|\frac{\left(t-\alpha_{i n}\right)\left(z-\beta_{i n}\right)}{\left(z-\alpha_{i n}\right)\left(t-\beta_{i n}\right)}\right|<P<1,
$$


then we also have for $n$ sufficiently large

(12.7) $\left|\frac{\left(z-\beta_{1}\right)\left(z-\beta_{2}\right) \cdots\left(z-\beta_{n+1}\right)\left(t-\alpha_{1}\right)\left(t-\alpha_{2}\right) \cdots\left(t-\alpha_{n}\right)}{\left(z-\alpha_{1}\right)\left(z-\alpha_{2}\right) \cdots\left(z-\alpha_{n}\right)(t-z)\left(t-\beta_{1}\right)\left(t-\beta_{2}\right) \cdots\left(t-\beta_{n+1}\right)}\right|<P_{1}{ }^{n}<1$,

for $\left(z-\beta_{n+1}\right) /\left((t-z)\left(t-\beta_{n+1}\right)\right)$ is uniformly bounded. It follows that $f_{n}(z)$ approaches $f(z)$ uniformly for $z$ in $R_{2}$. Moreover, if (12.6) holds for every $t$ on $C^{\prime}$ (bounding a closed region of analyticity of $f(z)$ which contains $R_{2}$ ), for every $z$ in an arbitrary closed region $R_{3}$ and for every $\alpha_{\text {in }}$ and $\beta_{\text {in }}$ in $R_{1}$ and $R_{2}$ respectively, then the sequence $f_{n}(z)$ converges uniformly to the function $f(z)$ for $z$ on $R_{3}$. This general result contains many of the various special theorems of the present paper. The uniform boundedness of $\left(z-\beta_{n+1}\right) /\left[(t-z)\left(t-\beta_{n+1}\right)\right]$ follows, it may be mentioned, from the fact that $R_{2}$ is interior to the region bounded by $C^{\prime}$, and this uniform boundedness follows even if no restriction of finiteness is made on the variables involved.

The general result just proved suggests the following problem:

Problem I. Let the closed regions $R_{1}, R_{2}, R_{3}$ be given. Determine a curve $C^{\prime}$ such that (12.6) is valid for $t$ on $C^{\prime}$ and for arbitrary points $\alpha_{i n}, \beta_{i n}, z$ in the given regions $R_{1}, R_{2}, R_{3}$ respectively.

It is to be noted that for convergence of $f_{n}(z)$ to $f(z)$ we require analyticity of $f(z)$ in the closed region containing $R_{2}$ and bounded by $C^{\prime}$, or more generally analyticity of $f(z)$ in the interior, continuity in the closed region.

Problem $I$ is equivalent to

Problem II. Let the closed regions $R_{1}, R_{2}, R_{3}$ be given. Find the locus $L$ of all points $t$ where $\alpha_{i n}, \beta_{i n}, z$ (varying independently) have $R_{1}, R_{2}, R_{3}$ as their respective loci, and the relation

$$
\left|\left(t, \alpha_{i n}, z, \beta_{\text {in }}\right)\right| \geqq 1
$$

obtains.

The set $L$ must of necessity contain $R_{2}$ and $R_{3}$, for if $t$ and $\beta_{\text {in }}$ coincide the left-hand member of (12.8) is infinite, and if $t$ and $z$ coincide the left-hand member of (12.8) is unity. The set $L$ is necessarily closed, since $R_{1}, R_{2}, R_{3}$ are closed. If the function $f(z)$ is analytic on the (closed) set $L$, and if $L$ does not contain the entire plane, then any curve $C^{\prime}$ in the complement of $L$ on which $f(z)$ is analytic is such that for $t$ on $C^{\prime}$ and for $\alpha_{i n}, \beta_{i n}, z$ arbitrary points in $R_{1}, R_{2}, R_{3}$, inequalities (12.6) and (12.7) are valid uniformly. The uniformity of (12.6) is an easy corollary of the closure of the sets $C^{\prime}, R_{1}, R_{2}, R_{3}$. Hence if $f(z)$ is analytic in the closed region bounded by $C^{\prime}$ which contains $L$, the sequence $f_{n}(z)$ converges uniformly to $f(z)$ for $z$ on $R_{3}$. 
We have supposed for convenience that $R_{1}, R_{2}, R_{3}$ are closed regions. If they are more general closed point sets, the remarks already made are valid if properly modified and interpreted. If $R_{1}, R_{2}, R_{3}$ are connected, then $L$ is also connected, but if one or more of those sets is not connected, then $L$ may fail to be connected, and it may be necessary to take $C^{\prime}$ as consisting of several distinct curves.

We have also assumed that the points $\alpha_{i n}$ and $\beta_{i n}$ lie on the closed point sets $R_{1}$ and $R_{2}$ respectively. It is, however, sufficient to assume that the points $\alpha_{\text {in }}$ have no limit point exterior to $R_{1}$ and that the points $\beta_{\text {in }}$ have no limit point exterior to $R_{\mathbf{2}}$. For under the new hypothesis, there exist auxiliary closed point sets $R_{1}^{\prime}$ and $R_{2}^{\prime}$ differing only slightly from but containing the point sets $R_{1}$ and $R_{2}$ respectively in their interiors. If the point sets $R_{1}{ }^{\prime}$ and $R_{2}{ }^{\prime}$ are suitably chosen, inequality (12.6) is still valid for suitable choice of $P$ for $t$ on $C^{\prime}$ and for $\alpha_{i n}, \beta_{i n}, z$ chosen arbitrarily in $R_{1}{ }^{\prime}, R_{2}{ }^{\prime}, R_{3}$; and the points $\alpha_{i n}, \beta_{\text {in }}$ lie in $R_{1}^{\prime}, R_{2}^{\prime}$ for $n$ sufficiently large.

Indeed, it is not even necessary to suppose that all limit points of the $\alpha_{i n}$ and $\beta_{i n}$ lie in $R_{1}$ and $R_{2}$. Let the $\alpha_{i n}$ be divided into two classes, $\alpha_{i n}{ }^{\prime}$ and $\alpha_{i n}{ }^{\prime \prime}$, where the former have all their limit points in $R_{1}$ and where the latter are for a given $n$ distinct from the $\beta_{i n}$ and have no limit point in $R_{3}$ but are otherwise unrestricted as to location, and where the number of the $\alpha_{i n}{ }^{\prime \prime}$ for a given $n$ is less than some $N$ independent of $n$. Let the $\beta_{\text {in }}$ be divided into two classes, $\beta_{i n}{ }^{\prime}$ and $\beta_{i n}{ }^{\prime \prime}$, where the former have all their limit points in $R_{2}$ and where the latter have no limit point exterior to $L$, and where the number of the $\beta_{i n}{ }^{\prime \prime}$ for a given $n$ is less than some $N$ independent of $n$. It is still true that if $f(z)$ is analytic in the closed region bounded by $C^{\prime}$ which contains $L$ then the sequence $f_{n}(z)$ converges uniformly to $f(z)$ for $z$ in $R_{3}$, for the expression

$$
\left|\frac{\left(z-\beta^{\prime \prime}\right)\left(t-\alpha^{\prime \prime}\right)}{\left(z-\alpha^{\prime \prime}\right)\left(t-\beta^{\prime \prime}\right)}\right|^{N}
$$

is uniformly bounded. The sets $\alpha_{i n}{ }^{\prime \prime}$ and $\beta_{i n}{ }^{\prime \prime}$ may even be allowed to be unlimited in number provided those points are suitably restricted, but the sufficient conditions here are more complicated and are left to the reader.

The cases we have been considering are under the assumption that the points $\alpha_{i n}$ and $\beta_{i n}$ are subjected generally to no heavier restriction than that of lying in $R_{1}$ and $R_{2}$ respectively or of having their limit points in those regions. If some or all of those points are suitably restricted in their respective regions, for instance so that at least $n / 2$ of them are independent of $i$, it may occur that our conclusion can be correspondingly broadened. But if those points $\alpha_{i n}$ and $\beta_{i n}$ are unrestricted except as indicated, the determination of 
$L$ as we have described it is the best determination of a region under the given hypothesis within which $f(z)$ must be analytic, for the sequence $f_{n}(z)$ to converge in the given $R_{3}$. For if $f(z)$ need not be analytic in the closed region $L$, the function $f(z)$ can be chosen to have a singularity at a point $t_{0}$ such that

$$
\left|\left(t_{0}, \alpha_{i n}, z_{0}, \beta_{i n}\right)\right| \geqq 1
$$

for a particular choice of $\alpha_{i n}, z_{0}, \beta_{i n}$ in $R_{1}, R_{3}, R_{2}$ respectively. Choose all the points $\alpha_{i n}$ and $\beta_{\text {in }}$ to coincide at these particular points and take for definiteness $\alpha_{i n}$ at infinity and $\beta_{i n}$ at the origin; this latter choice involves no loss of generality. Set $f(z)=1 /\left(z-t_{0}\right)$. We have

$$
\left|\left(t_{0}, \alpha_{i n}, z_{0}, \beta_{i n}\right)\right|=\left|\frac{\left(t_{0}-\alpha_{i n}\right)\left(z_{0}-\beta_{i n}\right)}{\left(z_{0}-\alpha_{i n}\right)\left(t_{0}-\beta_{i n}\right)}\right| \geqq 1,\left|z_{0}\right| \geqq\left|t_{0}\right| .
$$

The function $f_{n}(z)$ is the sum of the first $n$ terms of the Taylor expansion of $f(z)$ about the origin, and the sequence $f_{n}(z)$ diverges for the value $z=z_{0}$.

There are in reality four distinct problems connected with the regions (or other point sets) $R_{1}, R_{2}, R_{3}, L$, according as any three of these regions are given and the other is to be determined. We have mentioned in detail but a single problem, that in which $R_{1}, R_{2}, R_{3}$ are given; the others can be readily formulated and investigated by the reader. We shall consider more closely some special problems of these other types.

For instance, let $\alpha=R_{1}$ be the point at infinity, so that (12.8) reduces to

$$
\left|\frac{z-\beta_{i}}{t-\beta_{i}}\right| \geqq 1 \text {. }
$$

If the locus of $z$ is given as the closed interior of a circle $R_{3}$ and the locus of $t$ is given as a circumference $C^{\prime}$ which together with its interior has no point in common with $R_{3}$, then the locus of $\beta_{i}$ determined by (12.8) is an infinite closed region $B$ bounded by a branch of a certain hyperbola whose foci are the centers of $R_{3}$ and $C^{\prime}$. $^{*}$ Denote by $R_{2}^{\prime}$ the region complementary to $B$. Then if all points $\beta_{i}$ lie in a closed region $R_{2}$ interior to $R_{2}^{\prime}$, we have for arbitrary points $z, t$ of $R_{3}$ and $C^{\prime}$ respectively,

$$
\left|\frac{z-\beta_{i}}{t-\beta_{i}}\right|<P<1
$$

and it is readily shown that $P$ can be chosen independently of $\beta_{i}$, but depends on $R_{2}$. This inequality is the equivalent of (12.6), from which it follows that

* We omit the details of the proof, but they are entirely similar to those given by Walsh, American Mathematical Monthly, vol. 29 (1922), pp. 112-114. 
if $f(z)$ is analytic in the closed infinite region bounded by $C^{\prime}$, if the points $\alpha_{\text {in }}$ have no limit point other than the point at infinity, and if the points $\beta_{\text {in }}$ have no limit point exterior to $R_{2}$, then the sequence $f_{n}(z)$ converges to $f(z)$ uniformly for $z$ in $R_{3}$. We here use the fact mentioned above, that although (12.9) may not involve the actual cross ratios $\left(t, \alpha_{i n}, z, \beta_{i n}\right)$ of the points in (12.1), for points $\alpha_{i n}$ and $\beta_{i n}$ may lie exterior to $R_{1}$ (the point at infinity) and $R_{2}$, nevertheless (12.9) implies an inequality for $n$ sufficiently large of type (12.6) for the variables $t, \alpha_{i n}, z, \beta_{i n}$ which actually do occur in (12.1). The result we have just proved can be expressed in a form invariant under linear transformation.

The situation is similar if $\beta=R_{2}$ is the point at infinity, so that (12.8) reduces to $\left|\left(t-\alpha_{i}\right) /\left(z-\alpha_{i}\right)\right| \geqq 1$. If the locus of $z$ is given as the closed interior of a circle $R_{3}$ and if the locus of $t$ is given as a circumference $C^{\prime}$ which together with its interior has no point in common with $R_{3}$, the locus of $\alpha_{i}$ determined by (12.8) is an infinite closed region $R$ bounded by a branch of a hyperbola whose foci are the centers of $R_{3}$ and $C^{\prime}$. Denote by $R_{1}^{\prime}$ the region complementary to $R$. If all points $\alpha_{i}$ lie in a closed region $R_{1}$ interior to $R_{1}^{\prime}$, we have for arbitrary points $z, t$ of $R_{1}$ and $C^{\prime}$ respectively

$$
\left|\frac{t-\alpha_{i}}{z-\alpha_{i}}\right|<P<1,
$$

and it is readily shown that $P$ can be chosen independently of $\alpha_{i}$, but depends on $R_{1}$. It follows that if $f(z)$ is analytic in the closed infinite region bounded by $C^{\prime}$, if the points $\alpha_{i n}$ have no limit point exterior to $R_{1}$, and if the points $\beta_{\text {in }}$ have no limit point other than the point at infinity, then the sequence $f_{n}(z)$ converges to $f(z)$ uniformly for $z$ in $R_{3}$.

Problems I and II and the results connected with them are obviously formulated in a manner independent of linear transformation. Theorem $\mathrm{V}$ deals with the case that the regions $R_{1}, R_{2}, R_{3}$ are circular regions bounded by concentric circles, and the theorem following it is the generalization to the case that the circular regions $R_{1}, R_{2}, R_{3}$ are bounded by coaxial circles no two of which have a common point. It would be interesting, and is apparently an open problem, to solve Problem II for the case that $R_{1}, R_{2}, R_{3}$ are arbitrary circular regions. This problem has connections with some previous work by the present writer and others. ${ }^{*}$

If Problem II is considered for arbitrary regions $R_{1}, R_{2}, R_{3}$, but is so

* Walsh, these Transactions, vol. 22 (1921), pp. 101-116; vol. 23 (1922), pp. 67-88; Palermo Rendiconti, vol. 46 (1922). See also the reference given in $\$ 10$ of the present paper.

Coble, Bulletin of the American Mathematical Society, vol. 27 (1921), pp. 434-437.

Marden. these Transactions, vol. 32 (1930), pp. 81-109. 
modified that the right-hand member of (12.8) is replaced by a quantity less than unity, we are led to results on degree of convergence, and these are related to results on overconvergence.

The special case of Problems I and II where $R_{1}$ is the point at infinity, which thus includes interpolation by polynomials, has long been studied; interesting results are particularly due to Hermite, ${ }^{*}$ Runge, $\dagger$ Faber, $\ddagger$ and Jackson. $\S$ All the work on interpolation in the present paper is obviously related to Hermite's formula, which is (12.1) for the case $\alpha_{i n}=\infty$.

The special case in which $R_{1}$ is the point at infinity is quite simple; the locus $L$ consists of the closed interiors of all circles whose centers are points of $R_{2}$ and which pass through points of $R_{3}$. If If $R_{1}$ is the point at infinity and $R_{2}$ and $R_{3}$ coincide, the situation is still simpler, and the locus $L$ consists of the interior of every circle any one of whose radii is a segment joining two points of $R_{2}$. In the latter case, if $f(z)$ is analytic on the closed set $L$, then the sequence $f_{n}(z)$ converges uniformly to $f(z)$ for $z$ on $R_{2}$, where the points $\alpha_{i n}$ have no limit point except at infinity and the points $\beta_{\text {in }}$ have no limit point except on $R_{2}$. In particular, if $R_{2}$ is the interior of a circle, then $L$ is the interior of the concentric circle of three times the radius, as was proved by Jackson (loc. cit.); this case corresponds to Theorem $\mathrm{V}$ for $A=\infty, T=3 B$. If $R_{2}$ is a line segment, then $L$ is the interior of the two circles which have this segment as radius. A corollary of this is the following theorem:

Let the points $\beta_{\text {in }}$ be chosen arbitrarily in the interval $a \leqq z \leqq b$. If the function $f(z)$ is analytic for $|z-a| \leqq|a-b|$ and for $|z-b| \leqq|a-b|$, then the sequence of polynomials $f_{n}(z)$ of respective degrees $n$ which coincide with $f(z)$ in the respective points $\beta_{\text {in }}$ converges to the limit $f(z)$ uniformly for $a \leqq z \leqq b$.

Indeed, the reader will notice that the sequence $f_{n}(z)$ converges uniformly to $f(z)$ in some region containing the interval $a \leqq z \leqq b$ in its interior.

13. Poles at points $\left(A^{n}\right)^{1 / n}$. We have already considered interpolation in the $n$th roots of unity. As an analogous problem, we now study interpolation and approximation by rational functions whose poles are restricted to lie in the points $\left(A^{n}\right)^{1 / n}$, where $A>1$. Interpolation in the $n$th roots of unity is quite similar, as we have seen, to interpolation in the origin, and we shall

* Crelle's Journal, vol. 84 (1878), pp. 70-79.

$\dagger$ Theorie und Praxis der Reihen, Leipzig, 1904, pp. 126-142.

‡ Crelle's Journal, vol. 150 (1920), pp. 79-106.

Bulletin of the American Mathematical Society, vol. 34 (1928), pp. 56-63. The present results lead to a sharpening of the results of Jackson, even in the case of interpolation by rational functions no more general than polynomials.

I In particular, if $R_{1}$ is the point at infinity, and $R_{2}$ and $R_{3}$ are respectively $|z| \leqq r_{2},|z| \leqq r_{3}$, then $L$ is the region $|z| \leqq r_{2}+r_{3}$. This case, for interpolation by polynomials, was considered by Meray, Annales de l'Ecole Normale Supérieure, (3), vol. 1 (1884), pp. 165-176. 
find correspondingly that the properties of sequences of rational functions with poles in the points $\left(A^{n}\right)^{1 / n}$ are similar to the properties of sequences of polynomials. We shall not trouble to study explicitly rational functions whose poles are required to lie in the points $\left(A^{n}\right)^{1 / n}$ where $A$ is negative or imaginary, although such study involves only slight modifications of the formulas we use and only obvious modifications in the results we obtain.

If the function $f(z)$ is analytic for $|z|<T>1$, then the sequence $f_{n}(z)$ of rational functions of respective degrees $n$ whose poles lie in the points $\left(A^{n}\right)^{1 / n}, A>1$, of best approximation to $f(z)$ on $C:|z|=1$ in the sense of least squares converges to the limit $f(z)$ for $|z|<A, T$, uniformly for $|z| \leqq Z<A, T$.

The sequence $f_{n}(z)$ may, as we have already proved, be found by interpolation in the origin and in the points $\left(A^{n}\right)^{-1 / n}$, and the convergence of the sequence $f_{n}(z)$ to the function $f(z)$ depends on the approach to zero of the sequence

$$
\frac{z\left(A^{n} z^{n}-1\right)}{z^{n}-A^{n}} \frac{t^{n}-A^{n}}{t\left(A^{n} t^{n}-1\right)} .
$$

If $|z| \leqq Z<A, 1<Z, A<T^{\prime},|t|=T^{\prime}<T$, a sufficient condition for this approach to zero is

$$
\frac{A Z}{A} \frac{T^{\prime}}{A T^{\prime}}<1, \text { or } Z<A
$$

If $|z| \leqq Z<A, 1<Z, T \leqq A$, a sufficient condition is

$$
\frac{A Z}{A} \frac{A}{A T^{\prime}}<1 \text {, or } Z<T^{\prime} \text {. }
$$

The proof of the theorem is now complete.

The limit which we have found here, namely $Z<A, T$, can be replaced by no larger limit, as is seen by the illustration $f(z)=1 /(z-T)$. We have

$$
f_{n}(z)-f(z)=-\frac{\left(A^{n}-T^{n}\right) z\left(A^{n} z^{n}-1\right)}{T\left(1-A^{n} T^{n}\right)\left(z^{n}-A^{n}\right)(z-T)} .
$$

If $T>A$, this right-hand member fails to approach zero for $z=A$. If $T<A$, this right-hand member fails to approach zero for $z=T$. If $T=A$, all of the functions $f_{n}(z)$ naturally coincide with $f(z)$.

If $f(z)$ is analytic for $|z|<T>1$ and we consider the convergence for $|z| \leqq 1$ of the sequence of functions $f_{n}(z)$ of best approximation to $f(z)$ on $C$ in the sense of least squares whose poles lie in the points $\left(A_{n}^{n}\right)^{1 / n}$, where $A_{n}$ approaches unity as $n$ becomes infinite, then we always have uniform con- 
vergence for $|z| \leqq 1$ provided $A_{n}^{n}$ becomes infinite. The convergence of the sequence $f_{n}(z)$ depends on the approach to zero of

$$
\frac{z\left(A_{n}^{n} z^{n}-1\right)}{z^{n}-A_{n}^{n}} \frac{t^{n}-A_{n}^{n}}{t\left(A_{n}^{n} t^{n}-1\right)} \text {, or of } \frac{A_{n}^{n}+1}{A_{n}^{n}-1} \frac{T^{\prime n}}{A_{n}^{n} T^{\prime n}},
$$

so that $A_{n}^{n} \rightarrow \infty$ is sufficient, as is known from $\$ 7$. This condition cannot be lightened in the present case; we notice this by inspection of the illustration already given.

Let us denote by $\phi_{n}(z)$ the function of best approximation in the sense of least squares for the case of polynomial approximation; we compare the convergence of this sequence with the convergence of the sequence $f_{z}(z)$ previously considered, for best approximation in the sense of least squares when the poles $\left(A^{n}\right)^{1 / n}$ are prescribed. It is a remarkable phenomenon that if $T<A$ the difference $f_{n}(z)-\phi_{n}(z)$ approaches zero for $|z|<(A T)^{1 / 2}$, uniformly for $|z| \leqq Z<(A T)^{1 / 2}$, even though the function $f(z)$ may have singularities for $|z|=T$ or for $T<|z|<(A T)^{1 / 2}$. We can write

$$
f_{n}(z)-\phi_{n}(z)=\frac{1}{2 \pi i} \int_{C^{\prime}} \frac{f(t)}{t-z}\left[\frac{z\left(A^{n} z^{n}-1\right)\left(t^{n}-A^{n}\right)}{\left(z^{n}-A^{n}\right)\left(A^{n} t^{n}-1\right) t}-\frac{z^{n+1}}{t^{n+1}}\right] d t,
$$

where the integral is taken over $C^{\prime}:|z|=T^{\prime}<T$, and this expression approaches zero uniformly if that is true of the expression

$$
\frac{z\left(A^{n} z^{n}-1\right)\left(t^{n}-A^{n}\right)}{\left(z^{n}-A^{n}\right)\left(A^{n} t^{n}-1\right) t}-\frac{z^{n+1}}{t^{n+1}}=\frac{z\left(A^{n} t^{n} z^{n}+A^{n}-t^{n}-z^{n}\right)\left(t^{n}-z^{n}\right)}{\left(z^{n}-A^{n}\right) t^{n+1}\left(A^{n} t^{n}-1\right)}
$$

This last member approaches zero uniformly for $|z|=Z$ if $Z<\left(A T^{\prime}\right)^{1 / 2}$, so the result is established.

It will be noted that (13.1) is valid even if $|z|>T$, for the integrand considered as a function of $t$ has no singularity for $t=z$ and so represents the same analytic function (a rational function of $z$ of degree $n$ of form (2.1) plus a polynomial in $z$ of degree $n$ ) independently of the value of $z$.

It is not difficult to study the sequence $f_{n}(z)$ of rational functions of degree $n$ of best approximation to $f(z)$ on $C$ in the sense of least weighted $p$ th powers, whose poles lie in the points $\left(A^{n}\right)^{1 / n}, A>1$, but for the sake of simplicity we omit that discussion. Let us treat the question of interpolation in the points $\beta_{i n}$, where these points have no limit points of modulus greater than $B$.

If the function $f(z)$ is analytic for $|z|<T$, then the sequence $f_{n}(z)$ of rational functions of respective degrees $n$ with poles $\left(A^{n}\right)^{1 / n}$ found by interpolation in the points $\beta_{\text {in }}$, converges to the limit $f(z)$ uniformly for $|z| \leqq Z<(A T-A B-B T) / T$ if $T>A>B$, and uniformly for $|z| \leqq Z<T-2 B$ if $B<T<A$. 
A sufficient condition for the uniform convergence to zero of $f_{n}(z)-f(z)$ is the uniform convergence to zero of

$$
\frac{\left(z-\beta_{1}\right)\left(z-\beta_{2}\right) \cdots\left(z-\beta_{n+1}\right)\left(t^{n}-A^{n}\right)}{\left(z^{n}-A^{n}\right)\left(t-\beta_{1}\right)\left(t-\beta_{2}\right) \cdots\left(t-\beta_{n+1}\right)} .
$$

In the case $B<A<T$, it is sufficient if we have

$$
\frac{z+B}{A} \frac{T}{T-B}<1, \quad Z<\frac{A T-A B-B T}{T} .
$$

In the case $B<T<A$, it is sufficient if we have

$$
\frac{Z+B}{A} \frac{A}{T-B}<1, Z<T-2 B \text {. }
$$

These limits which we have found are the best possible limits, as is seen by considering the function $f(z)=1 /(z-T)$ and taking $\beta_{i n}=B$. We have

$$
f_{n}(z)-f(z)=\frac{\left(A^{n}-T^{n}\right)(z-B)^{n+1}}{(T-B)^{n+1}\left(z^{n}-A^{n}\right)(z-T)},
$$

which fails to approach zero for $z=-(A T-A B-B T) / T$ if $T>A$ and for $z=-T+2 B$ if $T<A$.

The case $B=0$ corresponds to interpolation in the origin or in points approaching the origin and is naturally not excluded in any of our discussion on interpolation in points $\beta_{i n}$. If $A>T$, the sequence $f_{n}(z)-\phi_{n}(z)$, where $f_{n}(z)$ is found by interpolation in the points $\beta_{\text {in }}$ and has its poles in the points $\left(A^{n}\right)^{1 / n}$ and $\phi_{n}(z)$ is the polynomial of interpolation in the points $\beta_{i n}$, may converge uniformly to the limit zero in a circle of radius larger than $T$. This phenomenon surely occurs if $B=0$ and also occurs for $B>0$ if $B$ is sufficiently small.

It is sufficient for $f_{n}(z)-\phi_{n}(z)$ uniformly to approach zero if the quantity

$$
\frac{\left(z-\beta_{1}\right)\left(z-\beta_{2}\right) \cdots\left(z-\beta_{n+1}\right)\left(t^{n}-z^{n}\right)}{\left(t-\beta_{1}\right)\left(t-\beta_{2}\right) \cdots\left(t-\beta_{n+1}\right)\left(z^{n}-A^{n}\right)}
$$

uniformly approaches zero, for which it is sufficient that $|z| \leqq Z,|t|=T^{\prime}<T$, $A>Z>T>B$,

$$
\frac{Z+B}{T^{\prime}-B} \cdot \frac{Z}{A}<1, \quad Z<\left(A T^{\prime}-A B+B^{2} / 4\right)^{1 / 2}-B / 2 .
$$

This quantity is greater than $T$ if $T^{\prime}$ is sufficiently near to $T$, and if $B$ is sufficiently small. The condition

$$
\left(A T-A B+B^{2} / 4\right)^{1 / 2}-B / 2>T
$$


can be written in the form

$$
A T>T^{2}+B T+A B,
$$

which is surely satisfied if $B=0$ and further if $B$ is small.

It is of secondary interest to study interpolation in the points $\left(B^{n}\right)^{1 / n}$ by rational functions with their poles in the points $\left(A^{n}\right)^{1 / n}$ and to compare the corresponding sequence of functions $f_{n}(z)$ with the sequence of functions $\phi_{n}(z)$ with poles at infinity or in the points $\left(A^{n}\right)^{1 / n}$ and found by interpolation in the points $\left(B^{n}\right)^{1 / n}$ or in the origin. This comparison presents no difficulty and is omitted. We remark however that if the two sequences $f_{n}(z)$ and $\phi_{n}(z)$ are found by interpolation in the points $\left(B^{n}\right)^{1 / n}$ and if the poles are respectively in the points $\left(A^{n}\right)^{1 / n}$ and at infinity, then under the assumption $B<T<A$, the sequence $f_{n}(z)-\phi_{n}(z)$ approaches zero uniformly for $|z| \leqq Z$ $<(A T)^{1 / 2}$. We remark too that interpolation by rational functions with poles in points on $|z|=A$ but not precisely the points $\left(A^{n}\right)^{1 / n}$, or even with poles in points near the points $\left(A^{n}\right)^{1 / n}$ but not on $|z|=A$, leads also to sequences of rational functions with properties similar to those of the corresponding sequence of polynomials; it would be of interest to determine the precise conditions on the new poles $\alpha_{i n}$ that this should be true.

The following is also an interesting problem, a possible generalization of the problem just suggested, and which can be solved at least in part by the methods we have used. Let the $\alpha_{i n}$ and $\beta_{i n}$ be given. What are the algebraic and geometric conditions on the $\alpha_{i n}{ }^{\prime}$ and $\beta_{i n}{ }^{\prime}$, such that the sequence $f_{n}(z)$ of rational functions of respective degrees $n$ whose poles lie in the points $\alpha_{\text {in }}$ which is found by interpolation in the points $\beta_{i n}$ should converge like the sequence $f_{n}^{\prime}(z)$ of rational functions of respective degrees $n$ whose poles lie in the points $\alpha_{i n}^{\prime}$ which is found by interpolation in the points $\beta_{i n}^{\prime}$, in the sense that for an arbitrary function $f(z)$ (satisfying certain restrictions) we have under suitable conditions $\lim _{n \rightarrow \infty}\left[f_{n}(z)-f_{n}^{\prime}(z)\right]=0$ uniformly?

14. More general approximation. There are some results not yet mentioned which follow directly from Theorem I. As an illustration we state

THEOREM VII. Let the function $f(z)$ be analytic in the interior of a Jordan region $J$, continuous in the corresponding closed region. If the region $J$ lies in a circular region $C$ and if the points $\alpha_{\text {in }}$ have no limit point in $C$, then there exists a sequence of functions $f_{n}(z)$ of the prescribed form (2.1) such that we have

$$
\lim _{n \rightarrow \infty} f_{n}(z)=f(z)
$$

uniformly in the closed region $J$.

Let $C$ be a finite region; this assumption involves no loss of generality. 
Let $f_{n}^{\prime}(z)$ represent the admissible function of degree $n$ of best approximation to $f(z)$ in (the closed region) $J$ in the sense of Tchebycheff; this function exists and is unique. If Theorem VII is not true, we have for some function $f(z)$, for some positive $\epsilon$, for some sequence $n_{k}$ of indices, and for some sequence of points $z_{k}$ belonging to the closed region $J$,

$$
\left|f_{n_{k}}^{\prime}\left(z_{k}\right)-f\left(z_{k}\right)\right|>\epsilon \text {; }
$$

we shall show that this leads to a contradiction. There exists* a polynomial $p(z)$ such that we have

$$
|f(z)-p(z)|<\epsilon / 2, \quad z \text { in } J .
$$

By Theorem I there exists a sequence of admissible functions $f_{n}(z)$ such that we have for $n_{k}$ sufficiently large

$$
\left|p(z)-f_{n_{k}}(z)\right|<\epsilon / 2, \quad z \text { in } C .
$$

These two inequalities yield

$$
\left|f_{n_{k}}(z)-f(z)\right|<\epsilon, \quad z \text { in } J,
$$

in contradiction with (14.2), assumed to hold for the function $f_{n_{k}}{ }^{\prime}(z)$ of degree $n_{k}$ of best approximation.

Perhaps it is worth while to state a more general theorem of wider applicability, of which Theorem VII is a special case.

Theorem VIII. Let $K^{\prime}$ and $K^{\prime \prime}$ be two classes of sequences of functions denoted generically by $\left\{f_{n}^{\prime}(z)\right\}$ and $\left\{f_{n}^{\prime \prime}(z)\right\}$, such that on a certain point set $K$ any function $f_{k}^{\prime \prime}(z)$ can be expressed as the limit of a uniformly convergent sequence $\left\{f_{n}^{\prime}(z)\right\}$. If $f(z)$ is an arbitrary function defined on $K$ which can be expressed as the limit of a uniformly convergent sequence $\left\{f_{n}^{\prime \prime}(z)\right\}$, then on $K$ the function $f(z)$ can also be expressed as the limit of a uniformly convergent sequence $\left\{f_{n}^{\prime}(z)\right\}$.

Theorem VIII is obvious in the case which frequently occurs, that the classes $f_{n}^{\prime}(z)$ and $f_{n}^{\prime \prime}(z)$ are defined as linear combinations of $n$ functions $\phi_{i}^{\prime}(z), \phi_{i}^{\prime \prime}(z)$, where $\phi_{i}^{\prime}(z)$ and $\phi_{i}^{\prime \prime}(z)$ do not depend on $n$, provided merely $i \leqq n$. In the more general case, the theorem is of interest, although the proof is similar to that of Theorem VII. In Theorem VIII the sequences $f_{n}^{\prime}(z)$ and $f_{n}^{\prime \prime}(z)$ are assumed to be independent of the functions represented - not to be found by interpolation or best approximation, or by any other requirement involving the limit function, and each function of the sequence is supposed to be independent of the others.

* Walsh, Mathematische Annalen, vol. 96 (1926), pp. 430-436. 
If Theorem VIII is not true, there exists some function $f(z)$ defined on $K$ which can be expressed on $K$ as the limit of a uniformly convergent sequence $f_{n}^{\prime \prime}(z)$ but such that $\delta_{n}$ does not approach zero, where $\delta_{n}$ is for each $n$ the greatest lower bound of the quantities

$$
\overline{\text { bound }}\left[\left|f(z)-f_{n}^{\prime}(z)\right|, z \text { on } K\right]
$$

for all admissible functions $f_{n}^{\prime}(z)$; the symbol bound indicates the least upper bound of all the quantities which follow. We shall show that this assumption leads to a contradiction.

Since the sequence $\delta_{n}$ does not approach zero, there exists a positive $\delta$ such that we have for an infinity of indices $n_{k}, \delta_{n_{k}}>\delta$. There exist, then, a sequence of indices $n_{k}$ such that we have

$$
\overline{\text { bound }}\left[\left|f(z)-f_{n_{k}}^{\prime}(z)\right|, z \text { on } K\right] \geqq \delta_{n_{k}}>\delta
$$

for all admissible functions $f_{n_{k}}{ }^{\prime}(z)$. There exists a function $f_{N}^{\prime \prime}(z)$ such that we have

$$
\left|f(z)-f_{N}^{\prime \prime}(z)\right|<\delta / 2, z \text { on } K
$$

There exists a sequence of admissible functions $f_{n}^{\prime}(z)$ such that we have for $n$ sufficiently large

$$
\left|f_{N}^{\prime \prime}(z)-f_{n}^{\prime}(z)\right|<\delta / 2, z \text { on } K \text {. }
$$

These two inequalities yield for $n=n_{k}$

$$
\left|f(z)-f_{n_{k}}^{\prime}(z)\right|<\delta, z \text { on } K,
$$

which is in contradiction with (14.3).

Theorem VII is a simple application of Theorem VIII. There are many other situations in which functions defined on more or less arbitrary point sets can be uniformly approximated by polynomials. * Each of these situations leads, by virtue of Theorem VIII, to a new result analogous to Theorem VII.

Theorem VII is not primarily concerned with the convergence to the function $f(z)$ of a particular set of functions $f_{n}(z)$, but if it is desired to have a uniquely determined set of such functions, the functions $f_{n}(z)$ of best approximation in the sense of Tchebycheff naturally furnishes such a set. Equation (14.1) is valid for this particular set, but we need not have the phenomenon of overconvergence. Theorem VIII is independent of the existence and uniqueness of functions $f_{n}^{\prime}(z), f_{n}^{\prime \prime}(z)$ of best approximation. 477-502.

* Seẹ for instance Walsh, these Transactions, vol. 30 (1928), pp. 472-482; vol. 31 (1929), pp.

These results lead easily to non-uniform expansions of arbitrary functions by rational functions of the form (2.1). 
The question may well be raised of the extension of Theorem $I$ to an arbitrary Jordan region $C$. We are not at present in a position to give a complete extension of Theorem I, but we can generalize Theorem VII:

THEOREM IX. If $C$ is an arbitrary Jordan region which contains no limit point of the set $\alpha_{i n}$, and if $f(z)$ is an arbitrary function analytic interior to $C$ and continuous in the corresponding closed region, then $f(z)$ can be expressed in the closed region $C$ as the limit of a uniformly convergent sequence of rational functions $f_{n}(z)$ of the form (2.1).

The proof is indirect. Assume the theorem not true; we shall reach a contradiction. There exists some function $f(z)$ of the kind described such that the sequence $\left\{f_{n}(z)\right\}$ of best approximation to $f(z)$ on $C$ in the sense of Tchebycheff does not converge uniformly to $f(z)$ on $C$. There exists some $\epsilon>0$ and some infinite sequence of indices $n_{k}$ such that for points $z_{n_{k}}$ in the closed region $C$ we have

$$
\left|f_{n_{k}}\left(z_{n_{k}}\right)-f\left(z_{n_{k}}\right)\right|>\epsilon \quad(k=1,2, \cdots) .
$$

Let us consider the points $\alpha_{i n_{k}}$. There exists some point $\alpha$, necessarily exterior to $C$, with the following property. For each neighborhood $\nu$ of $\alpha$ let $N_{k}^{(v)}$ denote the number of points $\alpha_{i n_{k}}$ in $\nu$ for that particular value of $k$. Then $\alpha$ is so to be determined that for some sequence of values of $k$ (say $k=k_{1}$, $k_{2}, \cdots$ ) the number $N_{k}^{(v)}$ becomes infinite no matter what neighborhood $\nu$ is chosen; the subsequence $k_{i}$ is thus to be independent of $\nu$. The proof is by subdivision of the plane, as in the proof of the Bolzano-Weierstrass theorem. Divide the plane into two half-planes $R_{1}$ and $R_{2}$. One or the other of these (closed) regions is a neighborhood $\nu$ such that for some subsequence $k_{1}^{\prime}, k_{2}^{\prime}, \cdots$ of the numbers $k$, the corresponding numbers $N_{k_{m}^{\prime}}^{(j)}$ become infinite as $m$ becomes infinite. Subdivide that region $R_{1}$ or $R_{2}$ or one of the regions $R_{1}$ and $R_{2}$ for which this fact holds. At least one of the new (closed) regions is a neighborhood $\nu$ such that for some subsequence $k_{1}^{\prime \prime}, k_{2}^{\prime \prime}, \cdots$ of the numbers $k_{i}^{\prime}$, the corresponding numbers $N_{k_{m}}^{(v)}$. become infinite as $m$ becomes infinite. It is no loss of generality here to choose $k_{1}^{\prime \prime}=k_{1}^{\prime}$. We continue subdivision of the plane in this way, so subdividing that the closed regions $\nu$ all contain some point $\alpha$ and that no point other than $\alpha$ is common to all the closed regions $\nu$. The next sequence $k_{m}^{\prime \prime \prime}$ of the numbers $k_{i}^{\prime \prime}$ is to be chosen so that $N_{k_{m}}^{(v)}$,' becomes infinite with $m$ and also so that $k_{1}^{\prime \prime \prime}=k_{1}^{\prime \prime}, k_{2}^{\prime \prime \prime}=k_{2}^{\prime \prime}$; similarly for the later sequences. Then by the diagonal process, choosing the numbers $k_{1}^{\prime}, k_{2}^{\prime \prime}, k_{3}^{\prime \prime \prime}, \cdots$, we obtain a subsequence $k_{1}, k_{2}, k_{3}, \cdots$ of numbers $k$ having the property desired.

We assume $\alpha$ to be the point at infinity; this can be brought about by a 
linear transformation of the complex variable. Choose as a neighborhood $\nu$ of $\alpha$ the exterior of a circle $\gamma$ which lies exterior to $C$. There exists a sequence $\lambda_{1}, \lambda_{2}, \lambda_{3}, \cdots$ of the numbers $k_{1}, k_{2}, k_{3}, \cdots$ such that $N_{\lambda_{j}}^{(\nu)} \geqq j$, where $\nu$ is now the exterior of $\gamma$. It follows from Theorem VII* that the sequence of functions $\left\{f_{n}(z)\right\}$ of best approximation to $f(z)$ on $C$ in the sense of Tchebycheff for the indices $n_{\lambda_{j}}$ approaches $f(z)$ uniformly in the closed region $C$; the number $n$ of Theorem VII is the present number $j$, and of the corresponding points $\alpha_{\text {in }}$ at least $j$ lie in $\nu$, but it is to be noted that a function $f_{n}(z)$ of form (2.1) with $j$ factors in the denominator is also a function $f_{n}(z)$ of form (2.1) with $n_{\lambda_{j}}$ factors in the denominator. Any increase in the number of possible factors (say from $j$ to $n_{\lambda_{j}}$ ) in the denominator of a function $f_{n}(z)$ of the form (2.1) with the corresponding increase in the degree of the function, can only decrease or at least not increase

$$
\max \left|f(z)-f_{n}(z)\right|, z \text { on } C,
$$

for the admissible function $f_{n}(z)$ of best approximation in the sense of Tchebycheff. The uniform convergence to $f(z)$ in the closed region $C$ of the functions $f_{n_{j}}(z)$ of best approximation as just proved by means of Theorem VII is in contradiction with (14.4), and the proof of Theorem IX is complete.

Theorem VIII now yields many new results on approximation when taken in conjunction with Theorem IX; see the references to the literature already given. For instance, if the function $f(z)$ is continuous on a Jordan $\operatorname{arc} C$ and if the points $\alpha_{i n}$ have no limit point on $C$, then there exists a sequence $f_{n}(z)$ of form (2.1) such that we have $\lim _{n \rightarrow \infty} f_{n}(z)=f(z)$ uniformly for $z$ on $C$.

The hypothesis in Theorem IX that the points $\alpha_{i n}$ have no limit point in the closed region $C$ cannot be replaced by the mere requirement that the points $\alpha_{\text {in }}$ lie exterior to $C$. We have already illustrated this fact by an example in $\$ 7$. If, however, we assume $f(z)$ merely analytic interior to the open Jordan region $C$ and that all the limit points of the $\alpha_{\text {in }}$ lie exterior to or on the boundary of $C$, there exists a sequence $\left\{f_{n}(z)\right\}$ such that we have $\lim _{n \rightarrow \infty} f_{n}(z)=f(z)$ for $z$ interior to $C$, uniformly for $z$ on any closed point set interior to $C$. Let $C_{1}, C_{2}, \cdots$ be a set of closed Jordan regions all interior to $C$, each with its boundary interior to its successor, and such that every point of $C$ lies in some $C_{k}$. The points $\alpha_{i n}$ have no limit point in the closed region $C_{k}$, so by Theorem IX there exist sequences $\left\{f_{n}^{(k)}(z)\right\}(k=1,2, \cdots)$ such that we have

$$
\lim _{n \rightarrow \infty} f_{n}^{(k)}(z)=f(z), \text { uniformly for } z \text { in } C_{k}, k=1,2, \cdots .
$$

Choose $N_{k}$ such that we have

* Theorem VII is by no means indispensable here, nor is Theorem I itself. The problem is easily reduced to one of approximation by polynomials. 


$$
\left|f_{n}^{(k)}(z)-f(z)\right|<1 / k, \text { for } z \text { in } C_{k}, n>N_{k},
$$

where we choose also $N_{k}>N_{k-1}$. Then the sequence

$$
\begin{gathered}
f_{1}^{\prime}(z), f_{2}^{\prime}(z), \cdots, f_{N_{2}}^{\prime}(z), f_{N_{2}+1}^{\prime \prime}(z), f_{N_{2}+2}^{\prime \prime}(z), \cdots, f_{N_{3}}^{\prime \prime}(z), f_{N_{3}+1}^{\prime \prime \prime}(z), \cdots, \\
f_{N_{4}}^{\prime \prime \prime}(z), f_{N_{4}+1}^{\text {iv }}(z), \cdots
\end{gathered}
$$

has the required property.

We remark that a suitable modification of the method just used yields direct proofs of Theorems VII and VIII.

It may be desired in Theorem IX (and similarly in other theorems) to approximate the given function in $C$ uniformly by rational functions $f_{n}(z)$ not merely of the form (2.1) but by rational functions $F_{n}(z)$ of the form (2.1) which effectively have poles at all of the assigned points $\alpha_{i n}$. This can naturally be accomplished. If the function $f_{n}(z)$ does not effectively have the poles $\alpha_{1 n}, \alpha_{2 n}, \cdots, \alpha_{k n}, k \leqq n$ (a point $\alpha_{i n}$ which is a $p$-fold zero of $f_{n}(z)$ is here to be enumerated $p+1$ times), we may set

$$
F_{n}(z)=\frac{\left(z-\alpha_{1 n}+\eta_{1 n}\right)\left(z-\alpha_{2 n}+\eta_{2 n}\right) \cdots\left(z-\alpha_{k n}+\eta_{k n}\right)}{\left(z-\alpha_{1 n}\right)\left(z-\alpha_{2 n}\right) \cdots\left(z-\alpha_{k n}\right)} f_{n}(z),
$$

where the numbers $\eta_{\text {in }}$ are positive and chosen sufficiently small, let us say so small that we have for $z$ on $C$

$$
\left|F_{n}(z)-f_{n}(z)\right|<\frac{1}{n} \text {. }
$$

Such a choice of the numbers $\eta_{i n}$ is always possible, for

$$
\lim _{\eta_{i n} \rightarrow 0} \frac{z-\alpha_{i n}+\eta_{i n}}{z-\alpha_{i n}}=1
$$

uniformly for $z$ on $C$. The replacing of the functions $f_{n}(z)$ by the functions $F_{n}(z)$ does not alter the character of the convergence of the original sequence, but it may naturally alter such minimum properties (for instance that of being rational functions of best approximation in some sense) as are possessed by the functions $f_{n}(z)$.

Theorem IX is equivalent to the statement that the sequence of rational functions $f_{n}(z)$ of best approximation to $f(z)$ on $C$ in the sense of Tchebycheff converges to the function $f(z)$ uniformly in the closed region $C$. This formulation can be generalized directly: 
THEOREM $\mathrm{X}$. Let $C$ be an arbitrary Jordan region which contains no limit point of the numbers $\alpha_{i n}$, and let $f(z)$ be an arbitrary function analytic interior to $C$ and continuous in the corresponding closed region. Then for the sequence $\left\{f_{n}(z)\right\}$ of rational functions (2.1) of best approximation to $f(z)$ on $C$, the measure of approximation of $f_{n}(z)$ to $f(z)$ on $C$ approaches zero with $1 / n$. If $\left\{F_{n}(z)\right\}$ is any sequence of functions analytic interior to $C$, continuous in the closed region, such that the measure of approximation of $F_{n}(z)$ to $f(z)$ on $C$ approaches zero with $1 / n$, and hence in particular for the sequence $\left\{f_{n}(z)\right\}$, we have

$$
\lim _{n \rightarrow \infty} F_{n}(z)=f(z),
$$

for $z$ interior to $C$, uniformly for $z$ on any closed point set interior to $C$.

The measure of approximation of $F_{n}(z)$ to $f(z)$ may here be taken as (1) $\max \left[n(z)\left|F_{n}(z)-f(z)\right|, z\right.$ on $\left.C\right]$, where $n(z)$ is continuous and positive on $C$; in this case (14.5) is valid uniformly for $z$ on $C$; $(2) \int n(z)\left|F_{n}(z)-f(z)\right|^{p}$ $|d z|, p>0$, where the integral is taken over the boundary (assumed rectifiable) of $C$; the function $n(z)$ is assumed continuous and positive on this boundary; (3) $\iint_{C} n(z)\left|F_{n}(z)-f(z)\right|^{p} d S, p>0$, where $n(z)$ is continuous and positive on $C$; (4) $\int_{\gamma} n(w)\left|F_{n}(z)-f(z)\right|^{p}|d w|, p>0$, where the interior of $C$ is mapped conformally onto the interior of the circle $\gamma:|w|=1$; the function $n(w)$ is assumed continuous and positive on $\gamma$. The proof of the fact that (14.5) is implied by the approach to zero of the measure of the approximation of $F_{n}(z)$ to $f(z)$, follows as in $\$ 8$. $^{*}$ The fact that for some sequence of functions $f_{n}(z)$ of form (2.1) the measure of approximation of $f_{n}(z)$ to $f(z)$ on $C$ approaches zero follows from Theorem IX, and this implies the approach to zero of the corresponding measure of approximation for the sequence of rational functions $f_{n}(z)$ of best approximation.

15. Further remarks. There are a number of variations of the problems already treated in detail, and some of these we shall mention.

1. It is natural to approximate the given function $f(z)$ analytic for $|z|<T>1$ by rational functions of type (2.1), and the approximation may be measured in the sense of least squares, by interpolation in the origin, by interpolation in certain roots of unity, together with the additional requirement of auxiliary conditions that we shall take as

$$
f_{n}\left(\beta_{k}\right)=f\left(\beta_{k}\right) \quad(k=1,2, \cdots, m)
$$

at $m$ arbitrarily chosen points $\beta_{k}$ interior to the circle $|z|=T$. The addition of requirement (15.1) does not essentially alter the results we have already

* Compare in connection with (4) also Walsh, these Transactions, vol. 32 (1930), pp. 794-816, and vol. 33 (1931), pp. 370-388. 
established, and involves only a slight modification in the proofs. The points $\beta_{k}$ are here considered limited in number, and may vary with $n$, although it is convenient to require $\left|\beta_{k}\right|<T^{\prime}<T$, where $T^{\prime}$ does not depend on $n$. Equation (15.1) is considered to involve various derivatives of the functions $f_{n}(z)$ and $f(z)$ if the points $\beta_{k}$ for a given $n$ are not all distinct.

This problem is considerably more difficult if the points $\beta_{k}$ are not limited in number, and has then close connections with our $\$ 12$. Interesting special cases have been studied in detail by Dunham Jackson (loc. cit.).

2. Another variation of the problems treated is to impose the additional requirement

$$
f_{n}\left(\beta_{k}\right)=B_{k} \quad(k=1,2, \cdots, m),
$$

for the approximating functions, where the points $\beta_{k}$ and quantities $B_{k}$ are now considered not to depend on $n$ and where the numbers $B_{k}$ have no necessary relation to the given function to be approximated. This new problem is different according to the original requirement made, best approximation in some sense or interpolation in some other given points. If we measure approximation on $C$ by the method of Tchebycheff, methods previously given by the present writer* apply here directly, even if $C$ is an arbitrary Jordan region, provided the points $\alpha_{\text {in }}$ have no limit point in the closed region. These methods apply also, in the case that $C$ is an arbitrary Jordan region and the points $\alpha_{i n}$ have no limit point in the closed region, for approximation in the sense of Tchebycheff to an arbitrary rational function with singularities interior to $C$. Overconvergence may take place in both of these situations. If $C$ is a circle and if the approximating functions $f_{n}(z)$ are found, in addition to (15.2), by interpolation at the origin, and if $\beta_{k} \neq 0$, the sequence $f_{n}(z)$ approaches the function $f(z)$ uniformly in some circle whose center is the origin. If another approximating sequence is found by interpolation in the roots of unity, in addition to (15.2), it would be an interesting problem, and not especially difficult, to determine the limit of the sequence $\left\{f_{n}(z)\right\}$, even if the given function has singularities interior to $C:|z|=1$.

3. Still another problem, analogous to the main problem of this paper, is to approximate a given function $f(z)$ analytic for $|z|<T>1$ by rational functions $f_{n}(z)$ of respective degrees $n$ whose zeros (instead of poles) are the prescribed points $\alpha_{i n},\left|\alpha_{i n}\right|>A>1$. The function $f(z)$ is to have no zero on or within $C:|z|=1$, for otherwise approximation on $C$ with an arbitrarily small error is impossible, by Hurwitz's theorem. We study the problem by taking up a different problem, that of approximating $1 / f(z)$ by the functions $1 / f_{n}(z)$,

* These Transactions, vol. 32 (1929), pp. 335-390. 
which we have already essentially considered. We have, if we measure approximation by least squares as in Theorem I,

$$
f_{n}(z)-f(z)=f(z) f_{n}(z)\left[\frac{1}{f(z)}-\frac{1}{f_{n}(z)}\right] .
$$

The square bracket approaches zero, the functions $f_{n}(z)$ are uniformly limited for $|z| \leqq 1$, so we have $\lim _{n \rightarrow \infty} f_{n}(z)=f(z)$ uniformly for $|z| \leqq 1$. If $f(z)$ has no zero and is analytic for $|z|<T$, we have $\lim _{n \rightarrow \infty} f_{n}(z)=f(z)$ uniformly for $|z|<R<\left(A T^{2}+T+2 A\right) /\left(A^{2}+2 A T+1\right)$. Further details may be worked out by the reader; the results we have stated are only the most obvious ones.

4. We raise still another question suggested by Theorem IX: can Theorem I including the result on overconvergence be extended to approximation in a region bounded by an arbitrary rectifiable Jordan curve? It may be noticed that in the various cases we have considered, interpolation in the points 0 , $1 / \bar{\alpha}_{k}$; in the origin; in the roots of unity; and in arbitrary points-our method is essentially that of expanding the function $1 /(t-z)$ in a sequence of rational functions of $z$ and $t$ found by the same conditions of interpolation as are prescribed for $f_{n}(z)$. For instance, in connection with (2.6) we have

$$
\frac{1}{t-z}=\lim _{n \rightarrow \infty}\left\{\frac{1}{t-z}\left[1-\frac{z\left(\bar{\alpha}_{1} z-1\right) \cdots\left(\bar{\alpha}_{n} z-1\right)\left(t-\alpha_{1}\right) \cdots\left(t-\alpha_{n}\right)}{\left(z-\alpha_{1}\right) \cdots\left(z-\alpha_{n}\right) t\left(\bar{\alpha}_{1} t-1\right) \cdots\left(\bar{\alpha}_{n} t-1\right)}\right]\right\}
$$

for suitable values of $z$ and $t$; the square bracket vanishes for $t=z$. The rational function whose limit is taken coincides with $1 /(t-z)$ for $z=0$ and $z=1 / \bar{\alpha}_{k}$. The only poles of these rational functions involving $z$ are the prescribed points $\alpha_{i n}$, and the expansion is valid under certain restrictions on $z$ and $t$. Termby-term integration of this sequence, when multiplied by the given function $f(t)$, over a suitably chosen path, yields a sequence of rational functions of $z$. converging to the limit function $f(z)$ under the conditions we have already determined. Presumably this same method, with suitable modifications, will apply in the more general case that $C$ is an arbitrary rectifiable Jordan curve. Results on approximation in the sense of Tchebycheff should be obtainable even if the Jordan curve is not rectifiable.

The study of analogous problems on approximation in multiply connected regions, where the $\alpha_{i n}$ are suitably distributed in the plane, should also be interesting.

5. We have considered in $\S \S 3,8$ the study of sequences of rational functions of respective degrees $n$ of best approximation whose poles are prescribed points $\alpha_{i n}$, having no limit point interior to the circle $|z|=A$. Our results yield at once new results on the sequences of rational functions of best approximation of respective degrees $n$ where the poles $\alpha_{i n}$ are not preassigned 
but are subject merely to the restriction $\left|\alpha_{i n}\right| \geqq A$. These new results are with reference to both degree of convergence and overconvergence. We remark incidentally that these new results as found by application of our results of $\S \S 3$, 8 are presumably not the most general results that can be obtained, but the problem appears to be a difficult one.

Another open problem is the study of the convergence of sequences of rational functions of respective degrees $n$ of best approximation where the poles are entirely without restriction.

6. In connection with the problem of approximation by rational functions, we remark that a particularly interesting case arises when the poles of the approximating functions are required to lie in the singularities of the function approximated.* It follows from the discussion of $\$ 13$ that if a function $f(z)$ is analytic interior to the circle $|z|=A>1$ but has this circle as a natural boundary, then the sequence of rational functions $f_{n}(z)$ of best approximation to $f(z)$ on $|z|=1$ in the sense of least squares, where the poles of the functions $f_{n}(z)$ lie in the points $\left(A^{n}\right)^{1 / n}$, converges to the function $f(z)$ uniformly for $|z| \leqq A^{\prime}<A$. A modification and amplification of that proof of $\$ 13$ shows that under the same hypothesis on $f(z)$ and under the same restriction of the poles of $f_{n}(z)$, the sequence $f_{n}(z)$ of rational functions of best approximation to $f(z)$ on any circle interior to $|z|=A$ in the sense of least weighted $p$ th powers, $p>0$, converges to the function $f(z)$ uniformly for $|z| \leqq A^{\prime}<A$.

7. The results of the present paper have almost immediate application to the study of approximation of harmonic functions by harmonic rational functions.

* Compare the paper by the writer in Acta Mathematica to which reference has already been made.

HARVARD UNIVERSITY,

Cambridge, Mass. 